ApJ, ACCepted (9/22/2011)

Preprint typeset using $\mathrm{L}^{A} \mathrm{~T} \mathrm{E}$ style emulateapj v. 11/10/09

\title{
A DISK AROUND THE PLANETARY-MASS COMPANION GSC 06214-00210 b: CLUES ABOUT THE FORMATION OF GAS GIANTS ON WIDE ORBITS*
}

\author{
Brendan P. Bowler, ${ }^{1,2}$ Michael C. Liu ${ }^{1}$ Adam L. Kraus,${ }^{1,3}$ Andrew W. Mann, ${ }^{1}$ \\ AND MiCHAEL J. IRELAND ${ }^{4,5}$ \\ ApJ, Accepted (9/22/2011)
}

\begin{abstract}
We present Keck/OSIRIS 1.1-1.8 $\mu \mathrm{m}$ adaptive optics integral field spectroscopy of the planetarymass companion to GSC 06214-00210, a member of the $~ 5$ Myr Upper Scorpius OB association. We infer a spectral type of $\mathrm{L} 0 \pm 1$, and our spectrum exhibits multiple signs of youth. The most notable feature is exceptionally strong $\mathrm{Pa} \beta$ emission $(E W=-11.4 \pm 0.3 \AA)$ which signals the presence of a circumplanetary accretion disk. The luminosity of GSC 06214-00210 b combined with its age yields a model-dependent mass of $14 \pm 2 M_{\mathrm{Jup}}$, making it the lowest-mass companion to show evidence of a disk. With a projected separation of $320 \mathrm{AU}$, the formation of GSC 06214-00210 b and other very lowmass companions on similarly wide orbits is unclear. One proposed mechanism is formation at close separations followed by planet-planet scattering to much larger orbits. Since that scenario involves a close encounter with another massive body, which is probably destructive to circumplanetary disks, it is unlikely that GSC 06214-00210 b underwent a scattering event in the past. This implies that planet-planet scattering is not solely responsible for the population of gas giants on wide orbits. More generally, the identification of disks around young planetary companions on wide orbits offers a novel method to constrain the formation pathway of these objects, which is otherwise notoriously difficult to do for individual systems. We also refine the spectral type of the primary from M1 to K7 and detect a mild $(2-\sigma)$ excess at $22 \mu \mathrm{m}$ using WISE photometry.
\end{abstract}

Subject headings: stars: pre-main sequence — stars: individual (GSC 06214-00210) - planetary systems: formation

\section{INTRODUCTION}

Direct imaging with adaptive optics on large telescopes is beginning to reveal the orbital architecture and demographics of extrasolar planetary systems on wide orbits (>10 AU). Several populations of planetary-mass companions are emerging in this nascent field, including giant planets residing in debris disks at moderate separations of $\lesssim 100$ AU (HR 8799 bcde: Marois et al. 2008, Marois et al. 2010; Fomalhaut b: Kalas et al. 2008; $\beta$ Pic b: Lagrange et al. 2009) and planetarymass objects on extremely wide orbits of several hundred AU (e.g., CHXR 73 B: Luhman et al. 2006; 1RXS J1609-2105 b: Lafrenière et al.|2008; Ross 458 C: Goldman et al. 2010, Scholz 2010).

Despite their growing numbers, the formation mechanisms of these companions remain obscure. Three plau-

\footnotetext{
bpbowler@ifa.hawaii.edu

${ }^{1}$ Institute for Astronomy, University of Hawai'i; 2680 Woodlawn Drive, Honolulu, HI 96822, USA

2 Visiting Astronomer at the Infrared Telescope Facility, which is operated by the University of Hawaii under Cooperative Agreement no. NNX-08AE38A with the National Aeronautics and Space Administration, Science Mission Directorate, Planetary Astronomy Program.

${ }^{3}$ Hubble Fellow.

${ }^{4}$ Department of Physics and Astronomy, Macquarie University, NSW 2109, Australia

${ }^{5}$ Australian Astronomical Observatory, PO Box 296, Epping, NSW 1710, Australia

* Some of the data presented herein were obtained at the W.M. Keck Observatory, which is operated as a scientific partnership among the California Institute of Technology, the University of California and the National Aeronautics and Space Administration. The Observatory was made possible by the generous financial support of the W.M. Keck Foundation.
}

sible (and non-mutually exclusive) routes have been proposed: core accretion plus gas capture (Pollack et al. 1996; Alibert et al. 2005), disk instability (Cameron 1978; Boss 1997), and direct collapse from molecular cloud fragmentation (Bate 2009). In situ formation through core accretion is unlikely for companions on wide orbits because the timescale to grow massive cores at these separations is longer than the observed lifetimes of protoplanetary disks ( $\sim 5 \mathrm{Myr}$; e.g., Hernández et al. 2007; Evans et al. 2009; Currie et al. 2009). On the other hand, models of disk instability have succeeded in forming gas giants between $\sim 20-100$ AU, with specific results depending on the initial conditions and physical assumptions of the simulations (Rafikov 2005; Stamatellos \& Whitworth 2008; Boss 2011).

There is debate about whether disk instability can account for planetary-mass companions at separations of several hundred AU (Boss 2006; Rafikov 2007; Nero \& Biorkman 2009; Stamatellos \& Whitworth 2009; Dodson-Robinson et al. 2009; Kratter et al.|2010; Rice et al. 2010; Boley et al. 2010; Baruteau et al. 2011). Some of the conflicting results arise from different approaches to modeling disk heating and cooling as well as uncertainties in the initial disk masses and surface densities, which are poorly constrained by observations during the embedded Class 0 and I protostellar phases when this mechanism is most likely to occur. Additionally, environmental factors such as envelope accretion onto the disk (both steady-state and episodic) are only beginning to be included in simulations (Vorobyov \& Basu 2010; Stamatellos et al. 2011). Despite these difficulties, there is some evidence that disks around Class 0 objects 
can be both massive $\left(>0.2 \mathrm{M}_{\odot}\right)$ and large $(>200 \mathrm{AU}$; Eisner et al. 2005; Jørgensen et al. 2005; Enoch et al. 2009; Jørgensen et al. 2009), leaving open the possibility that disk instability can form planets at wide separations.

There have been fewer theoretical studies focusing on the formation of gas giants by direct collapse from molecular clouds. In this scenario, planetary-mass objects form as ejected by-products of a fragmenting pre-stellar cloud core. Bate (2009) used numerical simulations to follow the gravitational collapse of a molecular cloud (see also Bate et al. 2002 and Bate et al. 2003) and found that all stellar and substellar objects begin as opacitylimited fragments with masses of a few times that of Jupiter and subsequently accrete gas, increasing their mass over time. In these simulations low-mass brown dwarfs and planetary-mass objects form directly from dense filamentary cloud structures and from instabilities in disks; in both cases they begin as dynamically unstable multiple systems and are ejected from dense regions of gas, halting strong accretion and limiting their masses below the hydrogen burning minimum mass. The formation of gas giants at extreme separations may have also proceeded in this fashion, perhaps by being ejected to wider orbits on faster timescales than brown dwarfs before appreciable accretion has occurred. Alternatively, as noted by Bate et al. (2003), preferential accretion from the primary could also explain the population of both brown dwarfs and planetary-mass companions on orbits of several hundred AU. In this framework of fragmentation plus ejection, planetary-mass objects on wide orbits represent the low-mass tail of brown dwarf companion formation.

In addition to these in situ formation models, other explanations for giant planets on wide orbits involve formation at close separations (perhaps through core accretion or disk instability) and subsequent orbital evolution to large separations. Several possibilities have been proposed, including outward scattering from dynamical interactions with another massive planet (Boss 2006; Debes \& Sigurdsson 2006; Scharf \& Menou 2009; Veras et al. 2009) and outward resonant migration with another planet while still embedded in a disk (Crida et al. 2009). These myriad possibilities make it difficult to identify the formation mechanisms of individual systems discovered by direct imaging.

The value of this population extends beyond informing formation scenarios; the direct detection of thermal photons enables detailed studies of their atmospheres. In particular, spectroscopy of young low-mass companions is providing insight into the influence of surface gravity on the atmospheric properties of low-temperature objects. Metchev \& Hillenbrand (2006) find that the young brown dwarf companion HD 203030 B has an earlier spectral type than expected from its evolutionary model-derived temperature, suggesting that gravity may impact the transition from L-type dwarfs to T-type dwarfs. More recent photometry and spectroscopy of the young ( $\sim 30 \mathrm{Myr}$; Zuckerman et al. 2011) HR 8799 planets indicate that they have unusually thick photospheric clouds, possibly signaling that thick clouds is a general phenomenon of young gas giants (Marois et al. 2008; Bowler et al. 2010; Currie et al. 2011; Madhusudhan et al. 2011; Barman et al. 2011a). The emergent spectrum of the $\sim 5$ Myr planetary-mass companion 2M1207b shows similar signs of a dusty atmosphere (Skemer et al. 2011; Barman et al. 2011b). There is a a growing need to explore this unexpected correlation with more objects spanning a range of gravities and temperatures (or equivalently, masses and ages).

Here we present near-infrared spectroscopy of the recently discovered planetary-mass companion GSC 0621400210 b (Kraus et al. 2008; Ireland et al. 2011), which orbits a member of the Upper Scorpius OB association $(\sim 5 \mathrm{Myr})$ at a projected separation of $320 \mathrm{AU}$. In $\S 2$ we present $J$ - and $H$-band spectroscopy of the companion as well as optical and near-infrared spectroscopy of the primary. We describe the spectroscopic properties of GSC 06214-00210 b, atmospheric model fits, predictions from evolutionary models, and analysis of the primary star in $\S 3$. In $\S 4$ we examine the validity of planetplanet scattering as a migration scenario for GSC 0621400210 b. Finally, $\S 5$ provides the conclusions of our work.

\section{OBSERVATIONS}

\subsection{Keck/OSIRIS J- and H-Band Spectroscopy of GSC 06214-00210 b}

We observed GSC 06214-00210 b on 9 July 2010 with the OH-Suppressing Infrared Imaging Spectrograph (OSIRIS; Larkin et al. 2006) integral field unit with natural guide star adaptive optics on Keck II. The weather was photometric with good seeing (0". $4-0$ "' 6 according to the DIMM on CFHT). We observed GSC 06214-00210 b in the $J b b(1.180-1.416 \mu \mathrm{m})$ and $H b b(1.473-1.803 \mu \mathrm{m})$ bandpasses with the $00^{\prime \prime} 05 \mathrm{pix}^{-1}$ plate scale, resulting in a lenslet geometry of $16 \times 64$, a field of view of $00^{\prime \prime} 8 \times 3^{\prime \prime} \cdot 2$, and a resolving power $(R \equiv \lambda / \delta \lambda)$ of $\sim 3800$. The separation of GSC 06214-00210 b from the primary $\left(2{ }^{\prime \prime} 2\right)$ is large compared to the measured FWHM of the companion $\left(0^{\prime \prime} 11\right.$ in $J b b ; 0^{\prime \prime} 13$ in $\left.H b b\right)$ and the seeing disk $\left(\sim 00^{\prime \prime} 5\right)$, so contamination from the primary is negligible. We obtained a total of $40 \mathrm{~min}$ in $J b b(5 \mathrm{~min} /$ exposure $\times 4$ nodded pairs) and $30 \mathrm{~min}$ in $H b b(5 \mathrm{~min} /$ exposure $\times 3$ nodded pairs) with $1^{\prime \prime}$ offsets along the long axis of the field of view. The airmass ranged from 1.351.64 during the observations. Immediately following our science observations we targeted the nearby A0V star HD 148968 at an airmass of 1.50-1.67. We also acquired sky frames in both filters before GSC 06214-00210 and after HD 148968.

The raw data were flat fielded, sky subtracted, cleaned for cosmic rays and bad pixels, assembled into 3D data cubes using the appropriate rectification matrices, and wavelength calibrated using version 2.3 of the OSIRIS Data Reduction Pipeline. Figure 1 shows an example of a collapsed $J$-band data cube. The science target and standard star spectra were extracted with aperture radii of 3 and 4 spaxels, respectively. The individual spectra from each band were then scaled to a common level and median combined. Spectral measurement uncertainties were determined by computing the standard errors about the median. The spectra were telluric corrected with the xtellcor general routine in the Spextool spectroscopic reduction package (Vacca et al. 2003; Cushing et al. 2004). We tested sky subtraction with sky frames (A-sky) and with the nodded science data (A-B) by reducing the data both ways; the influence on the final spectrum was minor. We chose the former $(\mathrm{A}-\mathrm{sky})$ 
for our final spectrum.

The $J$ - and $H$-band spectra were independently flux calibrated using flux ratios for GSC 06214-00210 from Ireland et al. (2011) together with 2MASS photometry of the primary (Skrutskie et al. 2006). Ireland et al. (2011) report flux ratios in the MKO system, so we use the 2MASS-MKO conversions from Leggett et al. (2006) to arrive at MKO apparent magnitudes for GSC 06214$00210 \mathrm{~b}$ of $J=16.25 \pm 0.04 \mathrm{mag}$ and $H=15.55 \pm 0.04 \mathrm{mag}$.

\subsection{IRTF/SpeX Near-Infrared Spectroscopy of the Primary GSC 06214-00210}

We observed the primary star GSC 06214-00210 with the SpeX spectrograph (Ravner et al. 2003) in short wavelength cross dispersed mode (SXD) at the NASA Infrared Telescope Facility (IRTF) on 29 April 2011 UT. The seeing reported by the CFHT DIMM was between $0{ }^{\prime \prime} 4-0.6$, and there were light cirrus clouds during the observations. We used the $0^{\prime \prime} 3$ slit aligned to the parallactic angle, yielding a resolving power of $\sim 2000$, and obtained a total of $4 \mathrm{~min}$ of data $(30 \mathrm{~s}$ /exposure $\times 4$ nodded pairs) by nodding along the slit in an ABBA pattern. After our science observations we targeted the nearby A0V star HD 144925 and obtained calibration frames at a similar airmass. The data were reduced with version 3.4 of Spextool (Vacca et al. 2003; Cushing et al. 2004).

We also acquired low-resolution $(R \sim 100)$ spectroscopy of the primary with IRTF/SpeX in prism mode on 12 May 2011 UT. The weather was poor with complete cirrus coverage so we used the opportunity to verify the spectral slope of our SXD spectrum. We used the 0".8 slit and obtained $160 \mathrm{~s}$ of data $(20 \mathrm{~s} /$ exposure $\times 4$ nodded pairs) at an airmass of 1.38. Immediately after we observed HD 144925 for telluric calibration.

\subsection{UH 2.2m/SNIFS Optical Spectroscopy of GSC 06214-00210}

We obtained an optical spectrum of GSC 06214-00210 on 16 May 2011 UT under clear conditions using the SNIFS instrument (Lantz et al. 2004) on the UH $2.2 \mathrm{~m}$ telescope. SNIFS is an optical integral field spectrograph with $R \sim 1000-1300$ that splits the signal with a dichroic mirror into blue $(\sim 3000-5200 \AA)$ and red ( $\sim 5200-9500 \AA)$ channels. The images are resampled with microlens arrays, dispersed with grisms, and focused onto blue- and red-sensitive CCDs.

A single $100 \mathrm{~s}$ exposure of the science target was sufficient to achieve high S/N ( 200). The processing was performed with the SNIFS data reduction pipeline, which is described in detail in Aldering et al. (2006) and Scalzo et al. (2010). The processing includes dark, bias, and flat-field corrections; assembling the data into red and blue 3D data cubes; cleaning them for cosmic rays and bad pixels; sky subtraction; extracting the spectra using a semi-analytic PSF model; and wavelengthcalibrating the spectra with arc lamp exposures taken at the same telescope pointing as the science data. Corrections for instrument response, airmass, and telluric lines are based on observations of the Feige 66 standard star using calibrated observations in Oke (1990). The far ends of the blue and red channels have low QE so to avoid these regions we trim our final spectrum to 3300
$4900 \AA$ and $5170-8700 \AA$.

\section{RESULTS}

\subsection{Properties of GSC 06214-00210 b}

3.1.1. Spectral Properties and Classification of GSC 06214-00210 b

Our OSIRIS spectrum of GSC 06214-00210 b is presented in Figure 2. The most striking feature in the data is the strong $\mathrm{Pa} \beta$ emission $(E W=-11.4 \pm 0.3 \AA)$ at $1.282 \mu \mathrm{m}$. The feature is present is each pair of dithered $J$-band observations of GSC 06214-00210 b and spans $\sim 9$ spectral elements (Figure 2, inset), so it is not a result of a cosmic ray or bad pixel. One of our $J$-band OSIRIS data sets include the primary (seen in Figure 1, the rest of data are dithered to avoid the star) and no $\mathrm{Pa} \beta$ emission is observed in that spectrum of the star. $\mathrm{Pa} \beta$ emission is also absent in our medium resolution SXD spectrum of the primary obtained $\sim 8.5$ months later. We therefore rule out the possibility that the emission observed in the companion is a result of contamination from the primary.

$\mathrm{Pa} \beta$ emission in young stars can arise from accretion or outflows (Folha \& Emerson 2001; Whelan et al. 2004), both of which imply the presence of a circumplanetary disk around GSC 06214-00210 b. The presence of a disk is bolstered by photometry from Ireland et al. (2011), who found a red $K-L^{\prime}$ color compared to field $\mathrm{M}$ and $\mathrm{L}$ dwarfs and suggest the excess may originate from thermal disk emission. Although other parameters such as metallicity, surface gravity, and dust can also affect the $K-L^{\prime}$ color of late-type objects, these are unlikely to be the origin of the red color for GSC 06214-00210 b based on the expected near solar-metallicity of USco members, a comparison to colors of field objects and giants (Ireland et al. 2011), and the minor influence of dust on $K-L^{\prime}$ colors at the $\mathrm{M} / \mathrm{L}$ transition predicted by models (Baraffe et al. 2003; Chabrier et al. 2000). We note that the $K-L^{\prime}$ color of $1.2 \mathrm{mag}$ is redder than many other young brown dwarfs with excesses attributed to disks (e.g., Liu et al. 2003; Allers et al. 2009). We describe the inferred accretion rate and luminosity in 3.1 .4 and we discuss the implications of a disk in the context of the formation of GSC 06214-00210 b in \$4.1

Other notable features in our spectrum include absorption at $\sim 1.20 \mu \mathrm{m}$ from $\mathrm{FeH}, 1.244$ and $1.253 \mu \mathrm{m} \mathrm{K} \mathrm{I}$ lines, deep $\mathrm{H}_{2} \mathrm{O}$ steam bands at $\sim 1.4$ and $\sim 1.8 \mu \mathrm{m}$, and $\mathrm{FeH}$ bandheads in the $H$ band near $1.6 \mu \mathrm{m}$ (Figure 2). All of these point to a late-M or early-L spectral type (Cushing et al. 2005).

Further classification of GSC 06214-00210 b warrants some care since there is no universally adopted nearinfrared classification scheme for young late-type stars and brown dwarfs. The classification of young brown dwarfs in the literature is inconsistent and often a mix of qualitative (comparative) and quantitative (index-based) systems (e.g., Reid et al. 1995; Kirkpatrick et al. 1999; Martín et al.|1999; /Reid et al.|2001; Geballe et al.|2002; Riddick et al. 2007); when multiple systems are used the adopted spectral type is usually a (subjectively weighted) average from several schemes.

We classify GSC 06214-00210 b primarily based on comparisons to near-infrared spectra of field objects and late-type free-floating members of Upper Scorpius (Figure 3). The advantage of using field objects is that they 
are optically typed and the advantage of using USco members is that they have the same age as GSC 0621400210 b. Spectra of field objects originate from the IRTF Spectral Library (Ravner et al. 2009) and those of USco objects from Lodieu et al. (2008). Many of the USco spectra have modest $\mathrm{S} / \mathrm{N}$ so we first Gaussian smoothed them from their native resolution of $R \sim 1700$ to $R \sim 1000$ and cleaned them for strong single pixel outliers probably originating form imperfect bad pixel or cosmic ray removal in the original reduction. For the comparative analysis in Figure 3 the OSIRIS spectrum of GSC 06214$00210 \mathrm{~b}$ was smoothed to the appropriate resolving power for each sample.

The K I lines in GSC 06214-00210 b are weaker than those in M8-L2 field objects, which is a common signature of youth (Gorlova et al. 2003; McGovern et al. 2004; Kirkpatrick et al. 2006). When normalized to $1.29-1.32 \mu \mathrm{m}$, as in Figure [3. the best matches to the $J$ band are M8-L0.5 dwarfs. The $J$-band in GSC 06214$00210 \mathrm{~b}$ is significantly bluer compared to the field L1 and L2 dwarfs. Together the joint $J+H$ spectra appear to best fit the L0.5 dwarf, although the 1.6-1.65 $\mu \mathrm{m} \mathrm{FeH}$ features are somewhat stronger in the field object.

The best USco matches are M9-L2 objects in the $J$ band and M9-L1 objects in the $H$ band. Interestingly, the $1.6 \mu \mathrm{m} \mathrm{FeH}$ features appear to be slightly stronger and the overall $H$ band less triangular in GSC 06214$00210 \mathrm{~b}$ compared to the USco members. The depth of the K I lines are similar for GSC 06214-00210 b and the M8-L2 sequence. Note that the spectral types of some objects from Lodieu et al. (2008) appear to be 1-3 subtypes later than their optical classifications (Herczeg et al. 2009; Biller et al. 2011).

Allers et al. (2007) define a spectral index from 1.50$1.57 \mu \mathrm{m}$ based on the depth of the $\mathrm{H}_{2} \mathrm{O}$ absorption that correlates well with optical spectral types from M5-L0 and is independent of gravity. This index yields a spectral type of M9.5 ${ }_{-1.1}^{+1.2}$ for GSC 06214-00210 b, which is consistent with the comparison to field objects and USco members. The errors incorporate spectral measurement uncertainties and intrinsic scatter in the relation. Altogether we assign GSC 06214-00210 b a spectral type of $\mathrm{L} 0 \pm 1$. Note that this agrees well with the estimate of M8-L4 by Ireland et al. (2011) based on colors.

\subsubsection{Effective Temperature and Bolometric Luminosity}

We fit the solar metallicity BT-Settl grid of model atmospheres (2010 version; Allard et al. 2010) to our spectrum of GSC 06214-00210 b. The BT-Settl models incorporate updated water opacity sources, revised solar abundances, and a new advanced treatment of dust formation using radiation hydrodynamic simulations. This new grid reproduces the observed colors and SEDs of late-M and $\mathrm{L}$ dwarfs better than the Ames-Cond and Ames-Dusty models of Allard et al. (2001), which examined the limiting cases of atmospheric dust formation. The grid spans effective temperatures between $2000-3000 \mathrm{~K}\left(\Delta T_{\text {eff }}=100 \mathrm{~K}\right)$ and gravities between 2.55.5 dex $(\operatorname{cgs} ; \Delta \log g=0.5)$. The fitting procedure relies on $\chi^{2}$ minimization as detailed in Cushing et al. (2008) and Bowler et al. (2009). To improve the $\mathrm{S} / \mathrm{N}$ of the data we Gaussian smoothed our OSIRIS spectrum from its native resolving power of $R \sim 3800$ to $R \sim 2000$ and propagated the measurement errors through the convolution. We smoothed the models to $R \sim 2000$ and fit them to the data in a Monte Carlo fashion by randomly generating synthetic observations based both on the spectral measurement uncertainties and the $J$ - and $H$-band photometric uncertainties (\$2.1) used for flux calibration. For each trial we determine the best-fitting spectrum and save the $\chi^{2}$ values from fitting every model in the grid. Figure 4 displays $\chi^{2}$ contour maps averaged over 100 Monte Carlo trials. Since the spectra are flux calibrated and the distance $(d)$ is constrained to $145 \pm 14$ pc (de Zeeuw et al. 1999; Ireland et al. 2011), the radius $(R)$ can be calculated from the multiplicative factor $\left(R^{2} / d^{2}\right)$ used to scale the models to the data (see Bowler et al. 2009). Figure 4 shows the contour plot of the inferred radius for GSC 06214-00210 b, representing averages from the Monte Carlo trials and incorporating the uncertainty in the distance estimate.

The $J(1.17-1.36 \mu \mathrm{m}), H(1.45-1.83 \mu \mathrm{m})$, and $J+H$ (1.17-1.83 $\mu \mathrm{m})$ spectral segments were fit separately. To avoid the $\mathrm{Pa} \beta$ emission line the $1.280-1.285 \mu \mathrm{m}$ region was excluded from the fits. The best-fitting model to the $J$ band is $T_{\text {eff }}=2700 \mathrm{~K} / \log g=4.5$ with $R=1.22 R_{\text {Jup }} .7$ The models match the depth of the $\mathrm{K}$ I lines and $1.35 \mu \mathrm{m} \mathrm{H}_{2} \mathrm{O}$ depth reasonably well, but they fail to reproduce the $1.2 \mu \mathrm{m} \mathrm{FeH}$ feature and the continuum level from $1.27-1.31 \mu \mathrm{m}$. In the $H$ band the models provide a better match the data. The best-fit model is $T_{\text {eff }}=2700 \mathrm{~K} / \log g=4.0$ with $R=1.36 R_{\mathrm{Jup}}$, which is similar to the result from the $J$ band.

A fit to the combined $J+H$ region produces a different result: $T_{\text {eff }}=3000 \mathrm{~K} / \log g=2.5$ and $R=1.02 R_{\mathrm{Jup}}$. The quality of the fit is rather poor as the $J$ band continuum slope deviates dramatically from the data and the depth of the K I lines are poorly reproduced. The $H$ band, however, is accurately reproduced by the model. In this case the best-fitting model is at the edge of the model grid so it may not represent the global minimum. The $2700 \mathrm{~K} / 4.0$ dex model that matches the individual bands overestimates the $J$-band flux and underestimates the $H$ band flux when fit to the entire spectrum.

Another approach to infer effective temperature is to use predictions from evolutionary models based on an object's age and bolometric luminosity. The age of the Upper Scorpius association has long been known to be $\sim 5 \mathrm{Myr}$, primarily constrained from fitting isochrones to known members in the HR diagram (see Preibisch \& Mamajek 2008; Slesnick et al. 2008). Inferring the age of a cluster from the HR diagram can be problematic, however, because pre-main sequence evolutionary models may have significant systematic errors (Hillenbrand \& White 2004; Kraus \& Hillenbrand 2009) perhaps caused in part by the influence of episodic accretion (Baraffe et al. 2009; Baraffe \& Chabrier 2010). With these uncertainties in mind, we adopt an age of $5 \pm 2$ Myr for GSC 06214-00210 b.

We calculated the bolometric luminosity by integrating an artificial spectrum constructed from the flux calibrated OSIRIS $J$ and $H$ band spectral segments combined with a model spectrum for wavelengths shorter than the $J$ band segment $(0.001-1.15 \mu \mathrm{m})$, between the

7 The standard value for Jupiter's radius is $71,492 \mathrm{~km}$ (Lindal et al. 1981), which is the equatorial radius at 1 bar. 
$J$ and $H$ segments $(1.36-1.45 \mu \mathrm{m})$, and longer than the $H$ band segment $(1.83-1000 \mu \mathrm{m})$. At each model-data interface the model was scaled to the data to create a continuous spectrum. Uncertainties in the spectra and flux calibration were accounted for in a Monte Carlo fashion. To test the sensitivity of the resulting luminosity on the input atmospheric model temperature we calculated the luminosity using three BT-Settl models with temperatures of $2000 \mathrm{~K}, 2500 \mathrm{~K}$, and $3000 \mathrm{~K}$, and a gravity of 4.0 dex. The influence on the resulting luminosity was negligible $(\sim 0.01 \mathrm{dex})$. Based on 1000 Monte Carlo trials we derived a bolometric luminosity of $-3.1 \pm 0.1$ dex for GSC 06214-00210 b.

To further verify our luminosity calculation we use the $K$-band bolometric correction calibrated with field objects from Golimowski et al. (2004). We arrive at a luminosity of $-3.04 \pm 0.10 \mathrm{dex}$, where the uncertainty takes into account errors in the distance, spectral type, photometry, and intrinsic scatter in the relation. In addition we compute the luminosity using parameters predicted from evolutionary models (see below; $\left.T_{\text {eff }}=2200 \mathrm{~K} / \log g=4.0\right)$. We flux calibrated the BTSettl, Ames-Dusty, and Ames-Cond (Allard et al. 2001) models to the mean of the $J$-, $H$-, and $K$-band flux calibration scaling factors, accounting for photometric uncertainties in a Monte Carlo fashion. The resulting luminosities are nearly identical at $-3.11 \pm 0.09$ dex, only differing by $\sim 0.01$ dex among the models. The radii inferred from the scaling factor and the distance (see above) are also virtually the same at $1.89 \pm 0.18 R_{\text {Jup }}$.

Figure [5] (top panels) displays interpolated temperature tracks as a function of luminosity and age for various evolutionary models. The left panel shows the Lyon Cond and Dusty models of Baraffe et al. (2003) and Chabrier et al. (2000), which demonstrate the limiting effects of photospheric dust formation (formation and settling vs. complete retention; Allard et al. 2001). The right panel shows the evolutionary models of Saumon \& Marlev (2008) for photospheres with no clouds ("nc") and those with significant amounts of dust $\left(" f_{\text {sed }}=2 "\right)$.

We determined effective temperatures in a Monte Carlo fashion by interpolating the evolutionary models. The Lyon models begin at 1 Myr and the Saumon \& Marley models begin at $3 \mathrm{Myr}$, so we excluded ages younger than those in the analysis. (The resulting PDFs for the age are truncated Gaussians in linear space). The Cond and Dusty models yielded nearly identical results of $2160 \pm 85 \mathrm{~K}$ for $10^{4}$ Monte Carlo draws. The Saumon \& Marley models were similar, yielding $2200 \pm 100 \mathrm{~K}$ for the case with no clouds and $2185 \pm 120 \mathrm{~K}$ for the cloudy version. These values are substantially lower than the those of the best-fitting model atmospheres.

The temperatures inferred from the atmospheric models $(\sim 2700-3000 \mathrm{~K})$ are warmer than previous determinations of young $\mathrm{M} / \mathrm{L}$ transition objects in the literature by 300-500 K (e.g., Béjar et al. 2008; Kuzuhara et al. 2011; Luhman 2004). A similar result was obtained by Dupuy et al. (2010) in their analysis of resolved late$\mathrm{M}$ binaries in the field with dynamical masses. They found that the most widely used atmospheric models systematically yield effective temperatures $\sim 250 \mathrm{~K}$ higher than the temperatures predicted by evolutionary mod- els, which are thought to be more reliable because they are less sensitive to missing or incomplete opacity sources (Chabrier et al. 2000). Likewise, the radii inferred from the atmospheric model fitting are systematically smaller than the value of $\sim 1.95 R_{\text {Jup }}$ predicted by evolutionary models (3.1.4), which is probably a result of overestimated effective temperatures in the fits.

Luhman (1999) developed an effective temperaturespectral type scale for young $M$ dwarfs intermediate between those of dwarfs and giants. The calibration was defined to ensure that components of the quadruple system GG Tau and members of the young cluster IC 348 were aligned on the same isochrone from the Baraffe et al. (1998) evolutionary models in the HR diagram. Luhman et al. (2003) revised the scale for M8 and M9 spectral types based on the latest-type members of IC 348 and Taurus. According to Luhman et al., the effective temperature of a young M9 object is $\sim 2400 \mathrm{~K}$. To estimate temperatures beyond the Luhman et al. scale, Allers et al. (2007) subtract relative offsets taken from the temperature scale of field objects. This amounts to $\sim 92 \pm 175 \mathrm{~K}$ for field M9-L0 objects based on the relation of Golimowski et al. (2004) (the uncertainty is from the rms scatter of the $S p T-T_{\text {eff }}$ relation). Assuming approximate uncertainties of $100 \mathrm{~K}$ for the Luhman et al. scale, this yields a temperature of $\sim 2310 \pm 200 \mathrm{~K}$, which is consistent with the evolutionary model predictions but disagree with those from the atmospheric model fitting.

$$
\text { 3.1.3. Mass }
$$

The mass of GSC 06214-00210 b was first estimated to be $\sim 12 M_{\text {Jup }}$ by Kraus et al. (2008) from $M_{K}$ measurements. It was recently updated to $\sim 12-15 M_{\text {Jup }}$ by Ireland et al. (2011) based on JHK colors and evolutionary models. We refine the mass determination using our luminosity measurement and a variety of evolutionary models (Figure [5, bottom panels). We use the same procedure to determine the mass from evolutionary models as we use in 33.1 .2 to determine temperature. The Cond and Dusty models yield $13.6 \pm 2.4 M_{\text {Jup }}$ and $14.2 \pm 2.4 M_{\text {Jup }}$, and the Saumon \& Marley cloudless and cloudy models yield $14.1 \pm 1.9 M_{\text {Jup }}$ and $14.4 \pm 1.8 M_{\text {Jup }}$. These values are in close agreement with previous estimates and hug the border of the brown dwarf/planetarymass limit as defined by the deuterium-burning limit ( 13 $M_{\text {Jup }}$; Spiegel et al. 2011).

\subsubsection{Accretion}

In a study of accretion diagnostics in young brown dwarfs, Natta et al. (2004) found that $\mathrm{Pa} \beta$ luminosity is well correlated with accretion luminosity at low masses. We use their empirical relation to derive the accretion luminosity and mass accretion rate for GSC 06214-00210 b.

The equivalent width of the $\mathrm{Pa} \beta$ emission line is $11.4 \pm 0.3 \AA$, and the flux from that line is $1.12 \pm 0.03 \times$ $10^{-18} \mathrm{~W} \mathrm{~m}^{-2}$. Assuming a distance of $145 \pm 14 \mathrm{pc}$ we find $\log \left(L_{\mathrm{Pa} \beta} / L_{\odot}\right)=-6.14 \pm 0.08$ for the $\mathrm{Pa} \beta$ line luminosity. Applying the empirical relationship between $\mathrm{Pa} \beta$ luminosity and accretion luminosity from Natta et al. (2004, Equation 2) yields an accretion luminosity of $\log \left(L_{\text {acc }} / L_{\odot}\right)=-4.4 \pm 1.3$. The mass accretion rate $(\dot{M})$ and the accretion luminosity are related through $\dot{M}=L_{\text {acc }} R / G M$, where $R$ is object's radius and $M$ is 
its mass. We calculate a radius from evolutionary models in the same fashion as in $\$ 3.1 .2$ and $\$ 3.1 .3$ and find $R=0.20 \pm 0.01 R_{\odot}$. This yields a mass accretion rate of $\log (\dot{M})=-10.7 \pm 1.3$, where $\dot{M}$ is in $M_{\odot} \mathrm{yr}^{-1}$. The uncertainty is dominated by scatter in the fitted relation from Natta et al. and reduces to 0.14 dex when these are ignored.

Mass accretion rates are observed to depend strongly on stellar mass and roughly follow an $\dot{M} \propto M_{*}^{2}$ empirical relationship (e.g., Muzerolle et al. 2005; Natta et al. 2006; although see Clarke \& Pringle 2006). This trend spans two orders of magnitude in mass and six orders of magnitude in mass accretion rate, although there are over two orders of magnitude of intrinsic scatter in the relation. Figure 6] shows the position of GSC 06214-00210 b in the $\log \dot{M}-\log M_{*}$ diagram relative to members of various star forming regions. The dependence of accretion rate on mass is clear, and objects with masses $\lesssim 30 M_{\text {Jup }}$ $\left(\log \left(M_{*} / M_{\odot}\right) \lesssim-1.5\right)$ appear to have accretion rates below $\sim 10^{-11} M_{\odot} \mathrm{yr}^{-1}$. GSC $06214-00210 \mathrm{~b}$ has a somewhat higher accretion rate than the lowest mass brown dwarfs from Muzerolle et al. (2005) and Herczeg et al. $(2009)$, although it is consistent with the scatter seen at higher masses.

While we have assumed the emission in GSC 06214$00210 \mathrm{~b}$ originates from energy released during accretion, we briefly examine whether it could result from chromospheric activity. Short \& Doyle (1998) modeled active $\mathrm{M}$ dwarf chromospheres and found that weak $\mathrm{Pa} \beta$ emission is possible at some chromospheric pressures. In Figure 7 we show the relative strength of the $\mathrm{Pa} \beta E W$ and line luminosity to objects from the Natta et al. (2006) sample of young stars. The $\mathrm{Pa} \beta$ emission strength for GSC 06214-00210 b is comparable to the strongest accretors from that sample and is larger than the vast majority of comparison stars. We can also examine the correlation between $\mathrm{Pa} \beta$ emission and the $\mathrm{H} \alpha 10 \%$ line width, which is a widely-adopted accretion indicator. Among the sample of young brown dwarfs from Natta et al. (2004), four out of the five that show evidence of accretion based on their $\mathrm{H} \alpha 10 \%$ widths also exhibit $\mathrm{Pa} \beta$ emission. No objects with $\mathrm{Pa} \beta$ emission were identified as non-accretors, which together with the unusually strong $\mathrm{Pa} \beta E W$ supports the notion that the observed emission in GSC 06214-00210 b is a result of accretion.

We note that GSC 06214-00210 b is also likely to have other emission lines. $\mathrm{H} \alpha$ is a good candidate since it correlates well with $\mathrm{Pa} \beta$ emission in brown dwarfs. $\mathrm{Br} \gamma$ is observed in some, but not all, accreting objects. For example, Natta et al. (2004) found that only two out of eight brown dwarfs with $\mathrm{Pa} \beta$ emission showed $\mathrm{Br} \gamma$ emission. However, $\operatorname{Br} \gamma$ emission does seem to correlate with the strength of the $\mathrm{Pa} \beta$ line, which increases the likelihood that GSC 06214-00210 b is emitting at that line.

\subsection{Properties of the Primary GSC 06214-00210}

The primary star GSC 06214-00210 is a weak-lined $\mathrm{T}$ Tauri member of Upper Sco (see Ireland et al. 2011 for a review of the literature). Medium resolution optical spectroscopy by Preibisch et al. (1998) revealed weak $\mathrm{H} \alpha$ emission $(E W=-1.51 \AA)$ which can be attributed to chromospheric activity. Our 0.8-2.5 $\mu \mathrm{m}$ SXD spectrum is presented in Figure 8, no emission lines are present. Compared to late- $\mathrm{K}$ and early-M dwarfs from the IRTF spectral library (Rayner et al. 2009), as shown in Figure 9 (right panel), the near-infrared spectrum resembles late-K objects more closely than early-M objects. We verified that our SXD spectrum was properly reduced by obtaining a low-resolution spectrum with SpeX in prism mode during a night of poor weather conditions. The shapes of the spectra are virtually identical (lower left inset of Figure 8), verifying our SXD spectrum and calling into question the nominal spectral type of M1 originally assigned by Preibisch et al. (1998).

Overall, the optical spectrum is best matched by that of a K7 dwarf from the Pickles (1998) spectral library from $\sim 3300-8700 \AA$ (Figure 9, left panel) 8 The shape of the SED and the depth of the TiO absorption bands differ substantially from those of the M1 spectrum. We also infer a spectral type using the spectral indices defined by Reid et al. (1995), which measure the depth of various molecular absorption bands in the optical. Our value for the TiO5 index (0.916) yields a spectral type of K5.5. Since many of the optical absorption bands are gravity-sensitive, a slightly earlier spectral type is not unexpected using this index-based scheme. Similarly, the $\mathrm{CaH} 2$ index (0.908) indicates an effective temperature of $\sim 4200 \mathrm{~K}$ using the $S p T-T_{\text {eff }}$ relation from Woolf \& Wallerstein (2006). All of these diagnostics suggest a spectral type of late-K rather than early-M, so we revise the spectral type of GSC 06214-00210 from M1 to $\mathrm{K} 7 \pm 0.5$.

We determine the effective temperature of GSC 0621400210 by constructing its SED from 0.6-22 $\mu \mathrm{m}$ (Figure 10) and comparing it to the grid of solar metallicity Phoenix-Gaia model atmospheres (Brott \& Hauschildt 2005). The photometry originate from the Carlsberg Meridian Catalog 14 (CMC14) for $r^{\prime}$ band (Evans et al. 2002); the Deep Near-Infrared Southern Sky Survey (DENIS) for $i, J$, and $K_{S}$ bands (Epchtein et al. 1997); the Two Micron All Sky Survey (2MASS) for $J, H$, and $K_{S}$ bands (Skrutskie et al. 2006); and the WideField Infrared Survey Explorer (WISE) for 3.4, 4.6, 12, and $22 \mu \mathrm{m}$ bands (Wright et al. 2010). Zero point flux densities are from Stoughton et al. (2002) for CMC14, Fouqué et al. (2000) for DENIS, Rieke et al. (2008) for 2MASS, and Wright et al. (2010) for WISE. The models are flux calibrated using the mean scaling factor from the 2MASS $J, H$, and $K_{S}$ bands. We limit the comparison to models with $\log g=4.0$, which is the approximate surface gravity from evolutionary models for a K7 star at 5 Myr. The $r^{\prime}$, i, and $J$ bands carry the most weight in constraining the effective temperature because the SED turns over to the Rayleigh-Jeans tail at $\lambda \gtrsim 1.6 \mu \mathrm{m}(H$ band). The best match is the $4200 \mathrm{~K}$ model, although the $4100 \mathrm{~K}$ and $4300 \mathrm{~K}$ models provide decent fits. Warmer and cooler model temperatures begin to diverge from the photometry, so we assign an uncertainty of $150 \mathrm{~K}$ for the temperature.

The WISE $22 \mu \mathrm{m}$ photometry disagrees with the model in Figure 10, suggesting a slight excess for the primary. In Figure 11 we compare the position of GSC 06214-00210 to field K7 dwarfs and other USco

\footnotetext{
8 We use the modification to the MK classification scheme that assigns $\mathrm{K} 7$ as an intermediate type between K5 and M0.
} 
members in the $W 1-W 4$ vs $J-K_{S}$ diagram. To isolate a sample of field $\mathrm{K} 7$ dwarfs we used a compilation of the revised Hipparcos catalog (van Leeuwen 2007) generated by E. Mamajek (private communication) with spectral types and $V$-band magnitudes from the original catalog (Perryman et al. 1997). An absolute magnitude cut of $M_{V}>5$ was imposed to exclude giants. This yielded 272 K7 dwarfs, which we then fed into the WISE Preliminary Release Source Catalog query using a search radius of $10^{\prime \prime}$. (The PSF FWHM for WISE bandpasses range between $6-12^{\prime \prime}$.) Among the resulting detections, we kept only those with the best photometric quality flags in all bands ( $p h_{-} q u a l=$ "A" or "B"), the best contamination and confusion flags ( $c c_{-}$flags $=$"0000"), extended source flags consistent with a point source (ext_flg="0"), and stable variable flags $\left(v a r_{-} f l g<5\right)$. This produced 41 objects, which are plotted in Figure 11. We performed a similar search of the WISE Preliminary Cata$\log$ for known USco members from Preibisch \& Mamajek (2008). We queried around 251 USco members with GKM spectral types applying the same flags as above; 67 objects yielded reliable WISE detections. Field stars and some USco members have $W 1-W 4$ colors of $\sim 0.0 \pm 0.2 \mathrm{mag}$, although most USco objects show moderate to large excess in that color. The $W 1-W 4$ color of $0.78 \pm 0.27$ for GSC $06214-00210$ indicates a mild $(2-\sigma)$ excess compared to field $\mathrm{K} 7$ stars.

We calculate the luminosity of the primary by integrating the flux calibrated $4200 \mathrm{~K} / \log g=4.0$ synthetic spectrum, yielding $L_{*}=0.38 \pm 0.07 L_{\odot}$. The error budget is dominated by the uncertainty in the distance (the uncertainty in effective temperature contributes $\sim 0.01 \mathrm{dex}$ ). In Figure 12 we show the position of GSC 06214-00210 in the HR diagram. The pre-main sequence evolutionary models of Baraffe et al. (1998) for $L_{\mathrm{mix}}=H_{p}$ are displayed from 0.02-1.4 $M_{\odot}$ with isochrones from $1 \mathrm{Myr}$ to $1 \mathrm{Gyr}$ (black). We also include the $L_{\mathrm{mix}}=1.9 H_{p}$ $1 \mathrm{Myr}, 5 \mathrm{Myr}, 10 \mathrm{Myr}$, and $1 \mathrm{Gyr}$ isochrones for higher masses $\left(\geq 0.6 M_{\odot}\right)$. Gray circles show the positions of Upper Scorpius low- and intermediate-mass members in Preibisch \& Mamaiek (2008, Tables 1 and 2). The position of GSC 06214-00210 is consistent with the scatter exhibited by other Upper Scorpius members, although it sits at a somewhat older isochrone than the nominal $5 \mathrm{Myr}$ age of the complex. For the prescription in Figure 12 with $L_{\text {mix }}=H_{p}$, the isochronal age is $\sim 16 \mathrm{Myr}$ with a 1- $\sigma$ range of $13-25 \mathrm{Myr}$. For $L_{\mathrm{mix}}=1.9 H_{p}$, which is required to fit the Sun, the inferred age is $\sim 10 \mathrm{Myr}$ with a range of 6-16 Myr. Note that the isochronal age of lowmass USco members $(\sim 5 \mathrm{Myr})$ diverges from the cluster age based on the $L_{\mathrm{mix}}=H_{p}$ prescription $(\sim 10 \mathrm{Myr})$, and instead is more consistent with the age inferred from the $L_{\mathrm{mix}}=1.9 H_{p}$ grid.

We estimate the mass of GSC 06214-00210 from its position on the HR diagram together with predictions from pre-main sequence evolutionary models. The BCAH98 models imply a mass of $\sim 1.0 M_{\odot}$ (Figure 121). We also compare it to the evolutionary models of D'Antona \& Mazzitelli (1994), which yield a mass of $\sim 0.8 M_{\odot}$, and Palla \& Stahler (1999), which yield $\sim 0.9 M_{\odot}$. Given these moderate systematic differences, we adopt a mass of $0.9 \pm 0.1 M_{\odot}$. Note that this is significantly higher than the value of $0.6 \pm 0.1 M_{\odot}$ that has been cited in previous work using the later M1 spectral type.

\section{DISCUSSION}

\subsection{Did GSC 06214-00210 b Experience a Scattering Event?}

As described in 11, several explanations can account for the observed population of planetary-mass companions orbiting stars at several hundred AU. Unfortunately, these models make few unique or testable predictions, and there is considerable debate about formation scenarios in the literature. Here we focus on the viability of one explanation, planet-planet scattering, in the context of our results for GSC 06214-00210 b.

The formation of closely packed planetary systems with two or more giant planets naturally leads to dynamical interactions which can significantly alter the orbits of one or more components. These close encounters produce a wide range of outcomes including (but not limited to) collisions between planets, accretion onto the star, rearrangement to new stable or quasistable orbits, scattering to close separations, scattering to highly eccentric wide orbits, and complete ejection ("ionization"). There is growing observational evidence that scattering is an important phenomenon in extrasolar planetary systems. It has been invoked to explain the population of planets with high eccentricities found in radial velocity surveys (e.g., Rasio \& Ford 1996; Ford \& Rasio 2008; (Jurić \& Tremaine 2008), the distribution of Rossiter-McLaughlin spin-orbit measurements (Morton \& Johnson 2011), and, more recently, the possible abundance of unbound gas giants inferred from microlensing surveys (Sumi et al. 2011).

We examine a hypothetical scattering event for GSC 06214-00210 b by assuming it was formed much closer than its present location through conventional means (core accretion or disk instability) and was ejected to a large orbit through the gravitational interaction with another massive body. Monte Carlo simulations of scattering events show that, given unequal planet mass ratios, the lower-mass planets show a much stronger preference for outward scattering than the more massive components (Veras \& Armitage 2004; Ford \& Rasio 2008) with little dependency on which planet initially had the wider orbit. If GSC 06214-00210 b was ejected to its present location as a result of such an event then it is likely that another object at least as massive $\left(\sim 14 M_{\text {Jup }}\right)$ also formed in that system.

Ejections of planets onto wide orbits are not uncommon outcomes in simulations so the discovery of a planetary-mass object at $\sim 300 \mathrm{AU}$ is perhaps not surprising. The presence of a disk around GSC 06214$00210 \mathrm{~b}$, however, implies that if it underwent scattering then its disk was not destroyed during point of closest approach with the scatterer. To our knowledge there are no studies that specifically investigate the survivability of circumplanetary disks during scattering events. Here we qualitatively examine the two relevant length scalesthe separation at closest approach during a scattering event and the circumplanetary disk radius - to assess the likelihood of a disk surviving such an encounter.

The criterion for orbital stability between two coplanar planets on circular orbits was found by Gladman (1993) to be 


$$
\Delta_{\text {cr }} \geq 2 \sqrt{3} R_{\mathrm{H}, \mathrm{M}},
$$

where $\Delta=a_{2}-a_{1}$ is the difference between the initial semimajor axes of the planets and $R_{\mathrm{H}, \mathrm{M}}$ is the mutual Hill radius (Marchal \& Bozis 1982):

$$
R_{\mathrm{H}, \mathrm{M}}=\left(\frac{m_{1}+m_{2}}{3 M_{*}}\right)^{1 / 3} \frac{a_{1}+a_{2}}{2} .
$$

This radius defines the region in which the gravitational force between two bodies is larger than the force on them due to the star. The critical range for stability $\Delta_{\text {cr }}$ can be divided into an outer region encompassing weak interactions, which can result in simple rearrangements of the system architectures with new quasi-stable orbits (Ford 2001; Veras \& Armitage 2004), and an inner region where strong interactions occur, which is characterized by chaotic events made up of collisions and ejections. This boundary must be found empirically from Monte Carlo simulations (see Gladman 1993 for the empirical relation for equal mass planets).

To test the critical range for stability $\Delta_{\mathrm{cr}}$, Chambers et al. (1996) ran simulations of two interacting planets with various separations and confirmed that their orbits were stable for at least $10^{7} \mathrm{yr}$ when $\Delta>2 \sqrt{3} R_{\mathrm{H}, \mathrm{M}}$, and when $\Delta<2 \sqrt{3} R_{\mathrm{H}, \mathrm{M}}$ they eventually experienced a close encounter with a distance at closest approach of $<1 \quad R_{\mathrm{H}, \mathrm{M}}$. Although the planet masses in that study were smaller than mass scales for a GSC 06214-00210-like system, a similar study for $\sim$ Jovian mass planets by Marzari (2002) resulted in similar close approach scales of $<1 R_{\mathrm{H}, \mathrm{M}}$ and a large frequency of ejections. Most scattering studies focus on various outcomes as a function of the initial planet spacings without discussing the distances of closest approach from the simulations, which is the relevant length scale we are interested in here. The exception is for collisions, defined by various authors to be the outcome when $\Delta$ becomes less than about the sum of the planetary radii. Given the relatively large frequency of collisions in some simulations (e.g., Ford 2001 find that collision rates can reach tens of percent depending on the planet radii and semimajor axes), the distances of closest approach appear to be quite small, which is consistent with the separation of $<1 R_{\mathrm{H}, \mathrm{M}}$ found by Chambers et al. (1996) and Marzari (2002).

Circumplanetary disks are thought to form around young gas giant planets from accretion of circumstellar disk material once a planet has opened a gap in the protoplanetary disk (e.g., Lubow et al. 1999; Ward \& Canup 2010). In our Solar System the regular satellites of the giant planets are fossil records of these structures (Canup \& Ward 2002) and the excess optical emission from Fomalhaut $\mathrm{b}$ has been attributed to scattering from an enormous disk around that extrasolar planet (Kalas et al. 2008). The outer radii of these disks have been investigated by several authors using analytical arguments and hydrodynamical simulations. Quillen \& Trilling (1998), Avliffe \& Bate (2009), and Martin \& Lubow (2011) obtain similar results of $\sim 0.3$ $0.4 R_{\mathrm{H}}$, which roughly corresponds the centrifugal radius. (Note that the individual Hill radius $R_{H}$ is the limiting case of the mutual Hill radius $R_{H, M}$ when $m_{2}$ goes to zero and $a_{1}=a_{2}$. If $m_{2}=c m_{1}$ and $a_{1} \sim a_{2}$ then $R_{\mathrm{H}, \mathrm{M}}$ and $R_{\mathrm{H}}$ differ by a factor of $(1+c)^{1 / 3}$.)

Since Hill radii depend on the planet masses and semimajor axes, we consider the 1:1, 1:2, and 1:5 cases of planet mass ratios to better understand the relative magnitudes of the scales of disk disruption and scattering closest approach. For an equal-mass coplanar scattering event $\left(m_{2}=m_{1}\right)$, the maximum separation at which the disks will not be disrupted is $\left(r_{\text {disk } 1}+r_{\text {disk } 2}\right) \sim 0.7 R_{\mathrm{H}}$. Assuming $a_{1} \sim a_{2}$ during a close encounter, the conversion from mutual Hill radius to the point-particle Hill radius is a factor of 1.26 , so the approximate scale of closest approach is $\lesssim 1.26 R_{\mathrm{H}}$. For the case where $m_{2}=2 m_{1}$, the disk disruption scale is $\sim 0.8 R_{\mathrm{H}}$ and the distance of closest approach is $\lesssim 1.4 R_{\mathrm{H}}$. Likewise, when $m_{2}=5 m_{1}$, the disruption scale becomes $\sim 0.95 R_{\mathrm{H}}$ and the distance of closest approach is $\lesssim 1.8 R_{\mathrm{H}}$.

These results suggest that the disruption of circumplanetary disks may be common in scattering events, especially if the distance to closest approach is less than about one half of a mutual Hill radius. The degree to which a disk is affected by these interactions (ie, disk truncation vs. complete destruction) likely depends on the disk sizes, planet mass ratio, relative disk inclinations, relative planet speeds, and impact parameter. Nevertheless, we interpret the retention of the disk around GSC 06214-00210 b as evidence against a past scattering event, although detailed simulations are needed.

Further insight into the formation of wide, low-mass companions can be gleaned from the architectures of other low-mass ratio systems (see 4.2 ). In a two-planet scattering event involving an ejection, energy conservation requires the remaining bound planet to have a final semimajor axis greater than one-half of its initial semimajor axis (e.g., Ford \& Rasio 2008). Since none of the wide planetary-mass companions discovered so far have an observed scatterer present in the system, the scattering hypothesis appears unlikely, although the dearth of published detection limits for additional companions in these systems hampers a more quantitative assessment. The notable exception is deep AO imaging of the 1RXS J1609-2105 system by Lafrenière et al. (2010), and somewhat shallower AO imaging of the GSC 0621400210 system by Ireland et al. (2011).

Finally, we note that if GSC 06214-00210 b was scattered to a large orbit then we can constrain the final location of the hypothetical scatterer using the aperture masking detection limits from Kraus et al. (2008) combined with the direct imaging detection limits from Ireland et al. (2011). As shown in Figure 13, the detection limits exclude objects with the same mass as GSC 06214-00210 b at projected separations $\gtrsim 170 \mathrm{AU}$, $30 M_{\text {Jup }}$ objects (roughly twice the mass of GSC 06214$00210 \mathrm{~b}) \gtrsim 60 \mathrm{AU}$, and $40 M_{\mathrm{Jup}}$ objects $\gtrsim 5 \mathrm{AU}$.

\subsection{Accretion in Bound and Free-Floating Planetary-Mass Objects}

The discovery of strong accretion in GSC 06214$00210 \mathrm{~b}$ prompts the broader question of how common this phenomenon is in other objects. Although the census of young planetary-mass companions has been steadily growing over the past several years, the 
moderate-resolution spectra needed to detect infrared emission lines have only been obtained for a fraction of these objects.

Six companions with masses $\lesssim 20 \quad M_{\text {Jup }}$ and ages $\lesssim 10$ Myr are known: SR $12 \mathrm{C}$ (Kuzuhara et al. 2011), CHXR 73 B (Luhman et al. 2006), 1RXS J1609-2105 b (Lafrenière et al. 2008), DH Tau b (Itoh et al. 2005), CT Cha B (Schmidt et al.|2008), and GSC 06214-00210 b (note that the uncertainties in masses and ages can be rather large). We have ignored companions to young brown dwarfs (e.g., 2M1207 Ab) since the formation of those systems was probably different from that of stars and wide companions. Low-resolution spectra have been published for most of these companions, but moderate-resolution spectra have only been acquired for 1RXS J1609-2105 b (Lafrenière et al. 2008; Lafrenière et al. 2010), CT Cha B (Schmidt et al. 2008), and GSC 06214-00210 b (this work). CT Cha B and GSC 06214-00210 b both show $\mathrm{Pa} \beta$ emission, but there is no evidence of accretion or thermal disk emission out to $4 \mu \mathrm{m}$ for 1RXS J1609-2105 b.

Two out of three very low-mass companions therefore show evidence of a disk. The sample size is minute, but this could hint that a large fraction of these objects are accreting. Moderate resolution near-infrared spectroscopy of the remaining companions would help address this matter. Intriguingly, the low-mass $\left(\sim 25 \quad M_{\text {Jup }}\right)$ brown dwarf companion GQ Lup B also shows $\mathrm{Pa} \beta$ emission (Seifahrt et al. 2007; Schmidt et al. 2008; Lavigne et al. 2009), which may support this notion.

With a large enough sample size, the presence of accretion or thermal disk emission from young planetary-mass companions might be used to learn about their formation mechanisms. If this population formed like free-floating planetary-mass objects then they should both share similar physical properties such as disk frequencies. Such a result would be a sign of a common formation mechanism. In the future, a similar analysis might be applied to extrasolar planets found at close separations (e.g., $<100 \mathrm{AU})$ and those at wide separations ( $>100 \mathrm{AU})$ to test formation mechanisms, perhaps in the context of core accretion and disk instability.

\section{CONCLUSION}

Our $J$ - and $H$-band spectroscopy of GSC 06214$00210 \mathrm{~b}$ reveals it is a late-type object $(\mathrm{L} 0 \pm 1)$ with several signatures of youth, including very strong $\mathrm{Pa} \beta$ emission. The discovery of accretion in this object confirms the suggestion by Ireland et al. (2011) based on its $K-L^{\prime}$ color that it possesses a circumplanetary disk. The accretion rate of $10^{-10.7} M_{\odot} \mathrm{yr}^{-1}$ is higher than other objects with comparable masses but is consistent with the scatter in accretion rates at larger masses. Atmospheric model fits to our spectrum yield relatively warm temperatures (2700-3000 K), which disagrees with the cooler predictions from evolutionary models $(2200 \pm 100 \mathrm{~K})$. With our new luminosity measurement of $-3.1 \pm 0.1$ dex, we refine the predicted mass of GSC 06214-00210 b to $14 \pm 2 M_{\text {Jup }}$, making it the lowest-mass companion to harbor a disk. In addition, our optical and near-infrared spectroscopy of the primary indicate an earlier spectral type of $\mathrm{K} 7 \pm 0.5$ than previously reported. We revise the mass estimate of GSC $06214-00210$ to $0.9 \pm 0.1 M_{\odot}$ based on our updated temperature and luminosity. WISE photometry of the primary reveals a marginal $(2-\sigma)$ excess at $22 \mu \mathrm{m}$.

GSC 06214-00210 b is one of only a handful of known companions orbiting stars at several hundred AU with masses straddling the brown dwarf/planetary-mass limit. While we cannot unambiguously distinguish the formation mechanism of GSC 06214-00210 b, we suggest that planet-planet scattering is an unlikely explanation. The small distance at closest approach between giant planets in a scattering event intimates that circumplanetary disks are probably disrupted during such encounters. If this conclusion is bolstered by more detailed dynamical simulations then another explanation- perhaps in situ formation - must be relevant for the population of planetary-mass companions on wide orbits. This also implies that the young free-floating planetary-mass objects with disks are probably not scattered planets and instead represent the low-mass tail of brown dwarf formation.

We thank our anonymous referee for thorough and helpful comments; Mark Marley, Didier Saumon, and France Allard for making their models available; Nicolas Lodieu for providing us with the spectra of USco objects; Eric Ford, Matthew Payne, and Aaron Boley for productive discussions about planet scattering; Eric Mamajek for the cross-matched Hipparcos catalog; Jim Lyke for his support with OSIRIS; and Niall Deacon and Will Best for their assistance during the observations. BPB is grateful to Michael Cushing, John Johnson, and Nader Haghighipour for helpful discussions. BPB and MCL have been supported by NASA grant NNX11AC31G and NSF grant AST09-09222. ALK has been supported by NASA through Hubble Fellowship grant 51257.01 awarded by STScI, which is operated by AURA, INc., for NASA under contract NAS 5-26555. This research has made use of the NASA/ IPAC Infrared Science Archive, which is operated by the Jet Propulsion Laboratory, California Institute of Technology, under contract with the National Aeronautics and Space Administration. This publication makes use of data products from the Wide-field Infrared Survey Explorer, which is a joint project of the University of California, Los Angeles, and the Jet Propulsion Laboratory/California Institute of Technology, funded by the National Aeronautics and Space Administration. We utilized data products from the Two Micron All Sky Survey, which is a joint project of the University of Massachusetts and the Infrared Processing and Analysis Center/California Institute of Technology, funded by the National Aeronautics and Space Administration and the National Science Foundation. NASA's Astrophysics Data System Bibliographic Services together with the VizieR catalogue access tool and SIMBAD database operated at CDS, Strasbourg, France, were invaluable resources for this work. The DENIS project has been partly funded by the SCIENCE and the HCM plans of the European Commission under grants CT920791 and CT940627. It is supported by INSU, MEN and CNRS in France, by the State of Baden-Württemberg in Germany, by DGICYT in Spain, by CNR in Italy, by FFwFBWF in Austria, by FAPESP in Brazil, by OTKA grants F-4239 and F-013990 in Hungary, and by the ESO C\&EE grant A-04-046. Finally, mahalo nui loa to the kama'āina of Hawai'i for their sup- 
port of Keck and the Mauna Kea observatories. We are grateful to conduct observations from this mountain.
Facilities: Keck:II (OSIRIS), IRTF (SpeX), UH:2.2m (SNIFS)

\section{REFERENCES}

Aldering, G., et al. 2006, ApJ, 650, 510

Alibert, Y., Mordasini, C., Benz, W., \& Winisdoerffer, C. 2005, A\&A, 434, 343

Allard, F., Hauschildt, P. H., Alexander, D. R., Tamanai, A., \& Schweitzer, A. 2001, ApJ, 556, 357

Allard, F., Homeier, D., \& Freytag, B. 2010, arXiv/astro-ph:1011.5405

Allers, K. N., et al. 2007, ApJ, 657, 511

-. 2009, ApJ, 697, 824

Ayliffe, B. A., \& Bate, M. R. 2009, MNRAS, 397, 657

Baraffe, I., \& Chabrier, G. 2010, A\&A, 521, A44

Baraffe, I., Chabrier, G., Allard, F., \& Hauschildt, P. H. 1998, A\&A, 337, 403

Baraffe, I., Chabrier, G., Barman, T. S., Allard, F., \& Hauschildt, P. H. 2003, A\&A, 402, 701

Baraffe, I., Chabrier, G., \& Gallardo, J. 2009, ApJ, 702, L27

Barman, T. S., Macintosh, B., Konopacky, Q. M., \& Marois, C. 2011a, ApJ, 733, 65

-. 2011b, ApJ, 735, L39

Baruteau, C., Meru, F., \& Paardekooper, S.-J. 2011, MNRAS, 1086

Bate, M. R. 2009, MNRAS, 392, 590

Bate, M. R., Bonnell, I. A., \& Bromm, V. 2002, MNRAS, 332 L65

-. 2003, MNRAS, 339, 577

Béjar, V. J. S., Osorio, M. R. Z., Pérez-Garrido, A., Álvarez, C., Martín, E. L., Rebolo, R., Villó-Pérez, I., \& Díaz-Sánchez, A. 2008, ApJ, 673, L185

Biller, B., Allers, K., Liu, M., Close, L. M., \& Dupuy, T. 2011, ApJ, 730, 39

Boley, A. C., Hayfield, T., Mayer, L., \& Durisen, R. H. 2010, Icarus, 207, 509

Boss, A. P. 1997, Science, 276, 1836

-. 2006, ApJ, 637, L137

-. 2011, ApJ, 731, 74

Bowler, B. P., Liu, M. C., \& Cushing, M. C. 2009, ApJ, 706, 1114

Bowler, B. P., Liu, M. C., Dupuy, T. J., \& Cushing, M. C. 2010, ApJ, 723, 850

Brott, I., \& Hauschildt, P. H. 2005, Proc. Gaia Symp. "The Three-Dimensional Universe with Gaia" (ESA SP-576), held at the Obs. de Paris-Meudon, 576, 565

Cameron, A. G. W. 1978, Moon and the Planets, 18, 5

Canup, R. M., \& Ward, W. R. 2002, AJ, 124, 3404

Chabrier, G., Baraffe, I., Allard, F., \& Hauschildt, P. 2000, ApJ, 542,464

Chambers, J. E., Wetherill, G. W., \& Boss, A. P. 1996, Icarus, 119,261

Clarke, C. J., \& Pringle, J. E. 2006, MNRAS Letters, 370, L10

Crida, A., Masset, F., \& Morbidelli, A. 2009, ApJ, 705, L148

Currie, T., et al. 2011, ApJ, 729, 128

Currie, T., Lada, C. J., Plavchan, P., Robitaille, T. P., Irwin, J., \& Kenyon, S. J. 2009, ApJ, 698, 1

Cushing, M. C., et al. 2008, ApJ, 678, 1372

Cushing, M. C., Rayner, J. T., \& Vacca, W. D. 2005, ApJ, 623, 1115

Cushing, M. C., Vacca, W. D., \& Rayner, J. T. 2004, PASP, 116, 362

D’Antona, F., \& Mazzitelli, I. 1994, ApJSS, 90, 467

de Zeeuw, P. T., Hoogerwerf, R., de Bruijne, J. H. J., Brown, A. G. A., \& Blaauw, A. 1999, AJ, 117, 354

Debes, J. H., \& Sigurdsson, S. 2006, A\&A, 451, 351

Dodson-Robinson, S. E., Veras, D., Ford, E. B., \& Beichman, C. A. 2009, ApJ, 707, 79

Dupuy, T. J., Liu, M. C., Bowler, B. P., Cushing, M. C., Helling, C., Witte, S., \& Hauschildt, P. 2010, ApJ, 721, 1725

Eisner, J. A., Hillenbrand, L. A., Carpenter, J. M., \& Wolf, S. 2005, ApJ, 635, 396

Enoch, M. L., Corder, S., Dunham, M. M., \& Duchêne, G. 2009, ApJ, 707, 103

Epchtein, N., et al. 1997, Messenger, 87, 27

Evans, D. W., Irwin, M. J., \& Helmer, L. 2002, A\&A, 395, 347
Evans, N. J., et al. 2009, ApJSS, 181, 321

Folha, D. F. M., \& Emerson, J. P. 2001, A\&A, 365, 90

Ford, E. 2001, Icarus, 150, 303

Ford, E. B., \& Rasio, F. A. 2008, ApJ, 686, 621

Fouqué, P., et al. 2000, A\&AS, 141, 313

Geballe, T. R., et al. 2002, ApJ, 564, 466

Gladman, B. 1993, Icarus, 106, 247

Goldman, B., Marsat, S., Henning, T., Clemens, C., \& Greiner, J. 2010, MNRAS, 405, 1140

Golimowski, D. A., et al. 2004, AJ, 127, 3516

Gorlova, N. I., Meyer, M. R., Rieke, G. H., \& Liebert, J. 2003, ApJ, 593, 1074

Herczeg, G. J., Cruz, K. L., \& Hillenbrand, L. A. 2009, ApJ, 696, 1589

Hernández, J., et al. 2007, ApJ, 662, 1067

Hillenbrand, L. A., \& White, R. J. 2004, ApJ, 604, 741

Ireland, M. J., Kraus, A., Martinache, F., Law, N., \& Hillenbrand, L. A. 2011, ApJ, 726, 113

Itoh, Y., et al. 2005, ApJ, 620, 984

Jørgensen, J. K., Bourke, T. L., Myers, P. C., Schöier, F. L., Dishoeck, E. F. V., \& Wilner, D. J. 2005, ApJ, 632, 973

Jørgensen, J. K., van Dishoeck, E. F., Visser, R., Bourke, T. L., Wilner, D. J., Lommen, D., Hogerheijde, M. R., \& Myers, P. C. 2009, A\&A, 507, 861

Jurić, M., \& Tremaine, S. 2008, ApJ, 686, 603

Kalas, P., et al. 2008, Science, 322, 1345

Kirkpatrick, J. D., Barman, T. S., Burgasser, A. J., McGovern, M. R., McLean, I. S., Tinney, C. G., \& Lowrance, P. J. 2006, ApJ, 639, 1120

Kirkpatrick, J. D., et al. 1999, ApJ, 519, 802

Kratter, K. M., Murray-Clay, R. A., \& Youdin, A. N. 2010, ApJ, 710,1375

Kraus, A. L., \& Hillenbrand, L. A. 2009, ApJ, 704, 531

Kraus, A. L., Ireland, M. J., Martinache, F., \& Lloyd, J. P. 2008, ApJ, 679, 762

Kuzuhara, M., Tamura, M., Ishii, M., Kudo, T., Nishiyama, S., \& Kandori, R. 2011, AJ, 141, 119

Lafrenière, D., Jayawardhana, R., \& van Kerkwijk, M. H. 2008, ApJ, 689, L153

-. 2010, ApJ, 719, 497

Lagrange, A.-M., et al. 2009, A\&A, 493, L21

Lantz, B., et al. 2004, Optical Design and Engineering. Edited by Mazuray, 5249, 146

Larkin, J., et al. 2006, New Astronomy Reviews, 50, 362

Lavigne, J.-F., Doyon, R., Lafrenière, D., Marois, C., \& Barman, T. 2009, ApJ, 704, 1098

Leggett, S. K., et al. 2006, MNRAS, 373, 781

Lindal, G. F., et al. 1981, Journal of Geophysical Research, 86 8721

Liu, M. C., Najita, J., \& Tokunaga, A. T. 2003, ApJ, 585, 372

Lodieu, N., Hambly, N. C., Jameson, R. F., \& Hodgkin, S. T. 2008, MNRAS, 383, 1385

Lubow, S. H., Seibert, M., \& Artymowicz, P. 1999, ApJ, 526, 1001

Luhman, K. L. 1999, ApJ, 525, 466

-. 2004, ApJ, 617, 1216

Luhman, K. L., Stauffer, J. R., Muench, A. A., Rieke, G. H. Lada, E. A., Bouvier, J., \& Lada, C. J. 2003, ApJ, 593, 1093

Luhman, K. L., et al. 2006, ApJ, 649, 894

Madhusudhan, N., Burrows, A., \& Currie, T. 2011, ApJ, 737, 34

Marchal, C., \& Bozis, G. 1982, Celestial Mechanics, 26, 311

Marois, C., Macintosh, B., Barman, T., Zuckerman, B., Song, I., Patience, J., Lafreniere, D., \& Doyon, R. 2008, Science, 322, 1348

Marois, C., Zuckerman, B., Konopacky, Q. M., Macintosh, B., \& Barman, T. 2010, Nature, 468, 1080

Martín, E. L., Delfosse, X., Basri, G., Goldman, B., Forveille, T., \& Osorio, M. R. Z. 1999, AJ, 118, 2466

Martin, R. G., \& Lubow, S. H. 2011, MNRAS, 413, 1447

Marzari, F. 2002, Icarus, 156, 570

McGovern, M. R., Kirkpatrick, J. D., McLean, I. S., Burgasser, A. J., Prato, L., \& Lowrance, P. J. 2004, ApJ, 600, 1020 
Metchev, S. A., \& Hillenbrand, L. A. 2006, ApJ, 651, 1166

Morton, T. D., \& Johnson, J. A. 2011, ApJ, 729, 138

Muzerolle, J., Luhman, K. L., Briceño, C., Hartmann, L., \& Calvet, N. 2005, ApJ, 625, 906

Natta, A., Testi, L., Muzerolle, J., Randich, S., ComerN, F., \& Persi, P. 2004, A\&A, 424, 603

Natta, A., Testi, L., \& Randich, S. 2006, A\&A, 452, 245

Nero, D., \& Bjorkman, J. E. 2009, ApJL, 702, L163

Oke, J. B. 1990, AJ, 99, 1621

Palla, F., \& Stahler, S. W. 1999, ApJ, 525, 772

Perryman, M. A. C., et al. 1997, A\&A, 323, L49

Pickles, A. 1998, PASP, 110, 863

Pollack, J. B., Hubickyj, O., Bodenheimer, P., Lissauer, J. J., Podolak, M., \& Greenzweig, Y. 1996, Icarus, 124, 62

Preibisch, T., Guenther, E., Zinnecker, H., Sterzik, M., Frink, S., \& Roeser, S. 1998, A\&A, 333, 619

Preibisch, T., \& Mamajek, E. 2008, Handbook of Star Forming Regions, 235

Quillen, A. C., \& Trilling, D. E. 1998, ApJ, 508, 707

Rafikov, R. R. 2005, ApJ, 621, L69

-. 2007, ApJ, 662, 642

Rasio, F. A., \& Ford, E. B. 1996, Science, 274, 954

Rayner, J. T., Cushing, M. C., \& Vacca, W. D. 2009, ApJSS, 185, 289

Rayner, J. T., Toomey, D. W., Onaka, P. M., Denault, A. J., Stahlberger, W. E., Vacca, W. D., Cushing, M. C., \& Wang, S. 2003, PASP, 115, 362

Reid, I. N., Burgasser, A. J., Cruz, K. L., Kirkpatrick, J. D., \& Gizis, J. E. 2001, AJ, 121, 1710

Reid, I. N., Hawley, S. L., \& Gizis, J. E. 1995, AJ, 110, 1838

Rice, W. K. M., Mayo, J. H., \& Armitage, P. J. 2010, MNRAS, 402, 1740

Riddick, F. C., Roche, P. F., \& Lucas, P. W. 2007, MNRAS, 381, 1067

Rieke, G. H., et al. 2008, AJ, 135, 2245
Saumon, D., \& Marley, M. S. 2008, ApJ, 689, 1327

Scalzo, R. A., et al. 2010, ApJ, 713, 1073

Scharf, C., \& Menou, K. 2009, ApJL, 693, L113

Schmidt, T. O. B., Neuhäuser, R., Seifahrt, A., Vogt, N.,

Bedalov, A., Helling, C., Witte, S., \& Hauschildt, P. H. 2008, A\&A, 491, 311

Scholz, R.-D. 2010, A\&A, 515, A92

Seifahrt, A., Neuhäuser, R., \& Hauschildt, P. H. 2007, A\&A, 463, 309

Short, C. I., \& Doyle, J. G. 1998, A\&A, 331, L5

Skemer, A. J., Close, L. M., Szücs, L., Apai, D., Pascucci, I., \& Biller, B. A. 2011, ApJ, 732, 107

Skrutskie, M. F., et al. 2006, AJ, 131, 1163

Slesnick, C. L., Hillenbrand, L. A., \& Carpenter, J. M. 2008, ApJ, 688,377

Spiegel, D. S., Burrows, A., \& Milsom, J. A. 2011, ApJ, 727, 57

Stamatellos, D., \& Whitworth, A. P. 2008, A\&A, 480, 879

-. 2009, MNRAS, 392, 413

Stamatellos, D., Whitworth, A. P., \& Hubber, D. A. 2011, ApJ, 730,32

Stoughton, C., et al. 2002, AJ, 123, 485

Sumi, T., et al. 2011, Nature, 473, 349

Vacca, W. D., Cushing, M. C., \& Rayner, J. T. 2003, PASP, 115, 389

van Leeuwen, F. 2007, A\&A, 474, 653

Veras, D., \& Armitage, P. J. 2004, MNRAS, 347, 613

Veras, D., Crepp, J. R., \& Ford, E. B. 2009, ApJ, 696, 1600

Vorobyov, E. I., \& Basu, S. 2010, ApJ, 714, L133

Ward, W. R., \& Canup, R. M. 2010, AJ, 140, 1168

Whelan, E. T., Ray, T. P., \& Davis, C. J. 2004, A\&A, 417, 247

Woolf, V. M., \& Wallerstein, G. 2006, PASP, 118, 218

Wright, E. L., et al. 2010, AJ, 140, 1868

Zuckerman, B., Rhee, J. H., Song, I., \& Bessell, M. S. 2011, ApJ, 732,61 
TABLE 1

Properties of the Primary GSC 06214-00210

\begin{tabular}{lc}
\hline \multicolumn{1}{c}{ Property } & Value \\
\hline \multicolumn{2}{c}{ Observed } \\
\hline$S p T$ & K7 \pm 0.5 \\
$L_{\text {Bol }}$ & $0.38 \pm 0.07 L_{\odot}$ \\
$r^{\prime}($ CMC14) & $11.94 \mathrm{mag}$ \\
$i$ (DENIS) & $11.08 \pm 0.03 \mathrm{mag}$ \\
$J$ (DENIS) & $10.05 \pm 0.06 \mathrm{mag}$ \\
$K_{S}$ (DENIS) & $9.31 \pm 0.06 \mathrm{mag}$ \\
$J(2 \mathrm{MASS})$ & $9.998 \pm 0.027 \mathrm{mag}$ \\
$H(2 \mathrm{MASS})$ & $9.342 \pm 0.024 \mathrm{mag}$ \\
$K_{S}(2 \mathrm{MASS})$ & $9.152 \pm 0.021 \mathrm{mag}$ \\
$3.4 \mu \mathrm{m}$ (WISE) & $9.094 \pm 0.024 \mathrm{mag}$ \\
$4.6 \mu \mathrm{m}$ (WISE) & $9.111 \pm 0.021 \mathrm{mag}$ \\
$12 \mu \mathrm{m}$ (WISE) & $8.985 \pm 0.035 \mathrm{mag}$ \\
$22 \mu \mathrm{m}$ (WISE) & $8.315 \pm 0.271 \mathrm{mag}$ \\
\hline \multicolumn{2}{c}{ Estimated } \\
\hline Distance & $145 \pm 14 \mathrm{pc}$ \\
Age & $5 \pm 2 \mathrm{Myr}$ \\
Mass & $0.9 \pm 0.1 \mathrm{M} \odot$ \\
$T_{\text {eff }}$ & $4200 \pm 150 \mathrm{~K}$ \\
\multicolumn{2}{c}{} \\
\hline
\end{tabular}

TABLE 2

Properties of the Companion GSC 06214-00210 B

\begin{tabular}{|c|c|}
\hline Property & Value \\
\hline \multicolumn{2}{|c|}{ Observed } \\
\hline $\begin{array}{l}S p T \\
\text { Proj. Sep. } \\
E W(\mathrm{~Pa} \beta) \\
\log \left(L_{\mathrm{Bol}} / L_{\odot}\right) \\
J(\mathrm{MKO})^{\mathrm{a}} \\
H(\mathrm{MKO})^{\mathrm{a}} \\
K(\mathrm{MKO})^{\mathrm{a}, \mathrm{b}} \\
L^{\prime \mathrm{a}}\end{array}$ & $\begin{array}{c}\mathrm{L} 0 \pm 1 \\
2 . \prime 2(320 \pm 30 \mathrm{AU}) \\
-11.4 \pm 0.3 \AA \\
-3.1 \pm 0.1 \\
16.25 \pm 0.04 \mathrm{mag} \\
15.55 \pm 0.04 \mathrm{mag} \\
14.94 \pm 0.03 \mathrm{mag} \\
13.75 \pm 0.07 \mathrm{mag}\end{array}$ \\
\hline \multicolumn{2}{|c|}{ Estimated } \\
\hline $\begin{array}{l}\text { Mass (Evol.) } \\
T_{\text {eff }}(\text { Evol.) } \\
T_{\text {eff }}(\text { BT-Settl }) \\
\log \left(L(\mathrm{~Pa} \beta) / L_{\odot}\right) \\
\log \left(L_{\text {acc }} / L_{\odot}\right) \\
\log \left(\dot{M} / M_{\odot} \mathrm{yr}^{-1}\right)\end{array}$ & $\begin{array}{c}14 \pm 2 M_{\mathrm{Jup}} \\
2200 \pm 100 \mathrm{~K} \\
2700 \pm 200 \mathrm{~K} \\
-6.14 \pm 0.08 \\
-4.4 \pm 1.3 \\
-10.7 \pm 1.3\end{array}$ \\
\hline
\end{tabular}




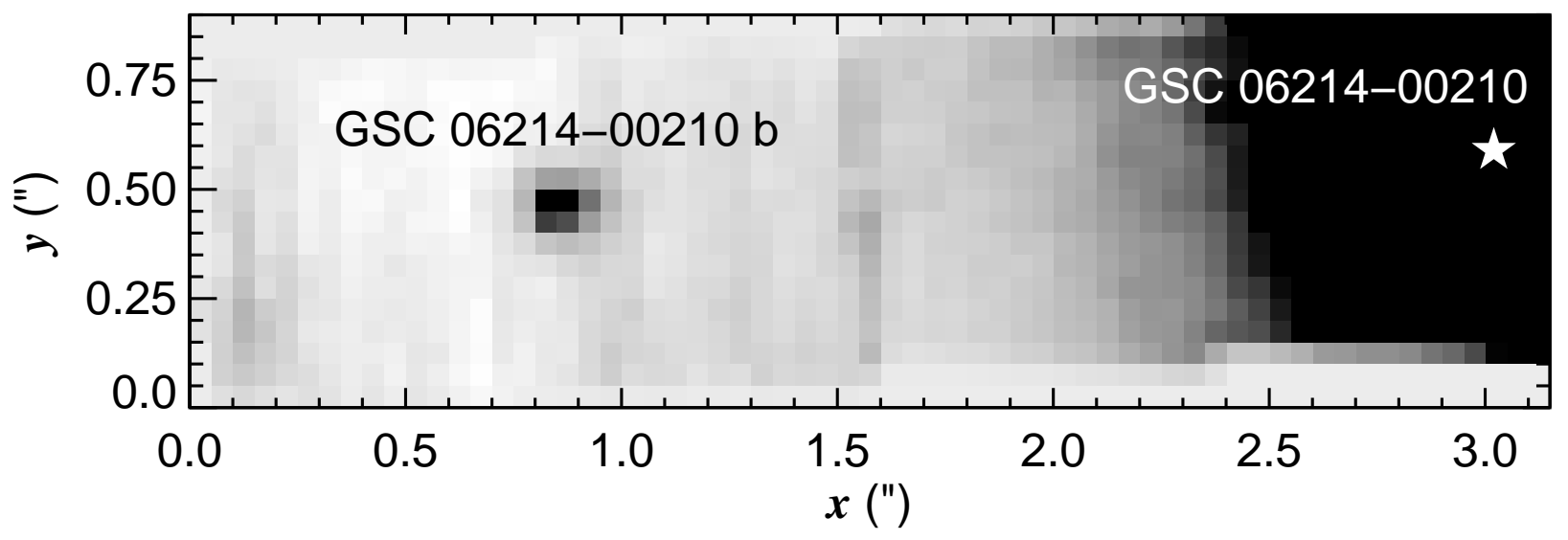

FIG. 1.- Median-collapsed $J$-band OSIRIS cube showing GSC 06214-00210 and its companion. The primary is marked with a white star. The spaxel scale is $0{ }^{\prime \prime} 05 \mathrm{pix}^{-1}$ and the separation between the primary and the companion is $2{ }^{\prime \prime} 2$. 


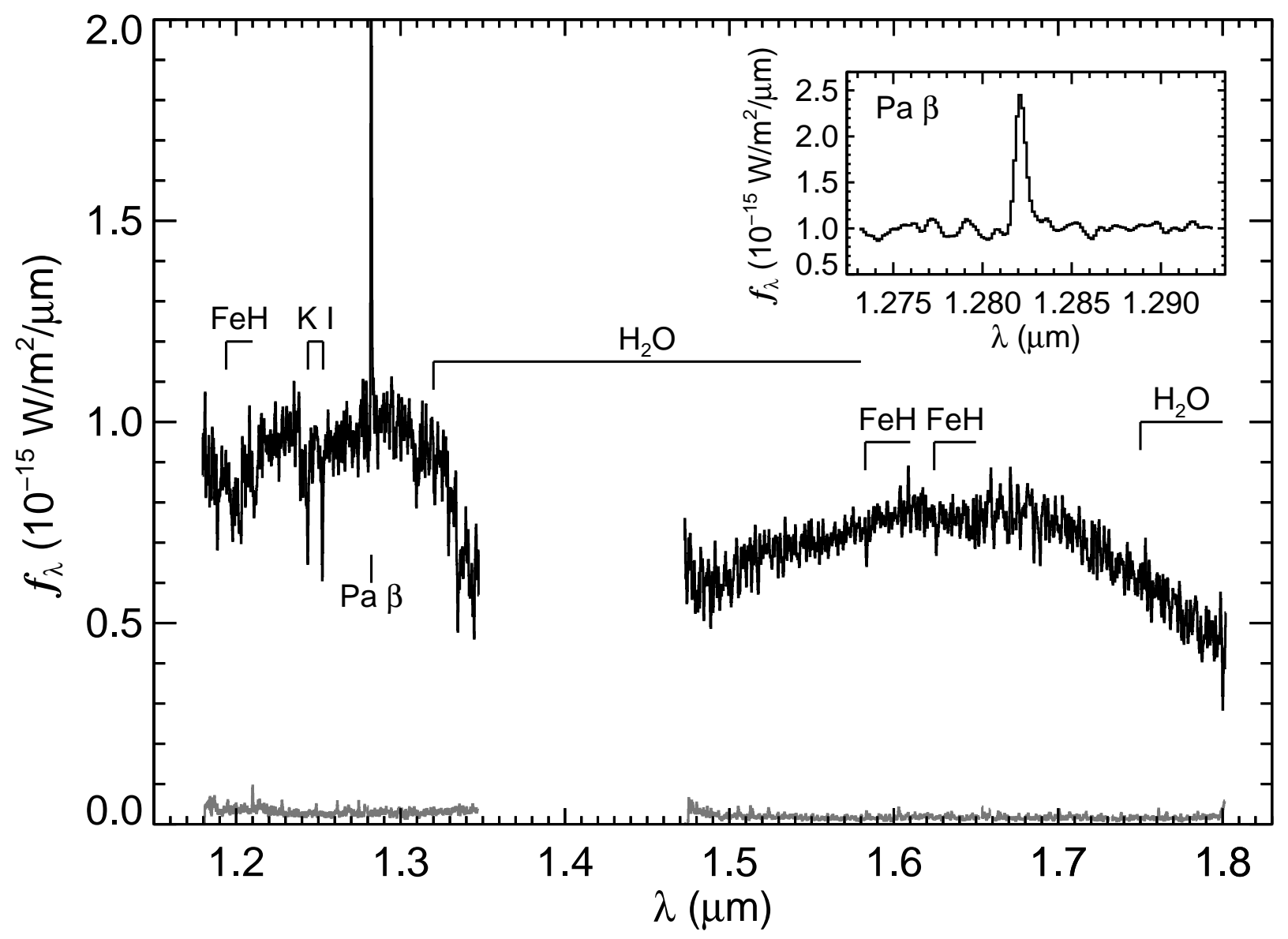

FIg. 2.- Flux-calibrated $J$ and $H$ band spectra of GSC $06214-00210 \mathrm{~b}$. The strong emission line at $1.282 \mu \mathrm{m}$ (inset) is Pa $\beta$ ( $E W=-$ $11.4 \pm 0.3 \AA$ ). The spectrum exhibits absorption features typical of late-M/early-L spectral types including FeH, $\mathrm{K} \mathrm{I}$, and $\mathrm{H}_{2} \mathrm{O}$. Spectral measurement uncertainties are shown at the bottom in gray. 

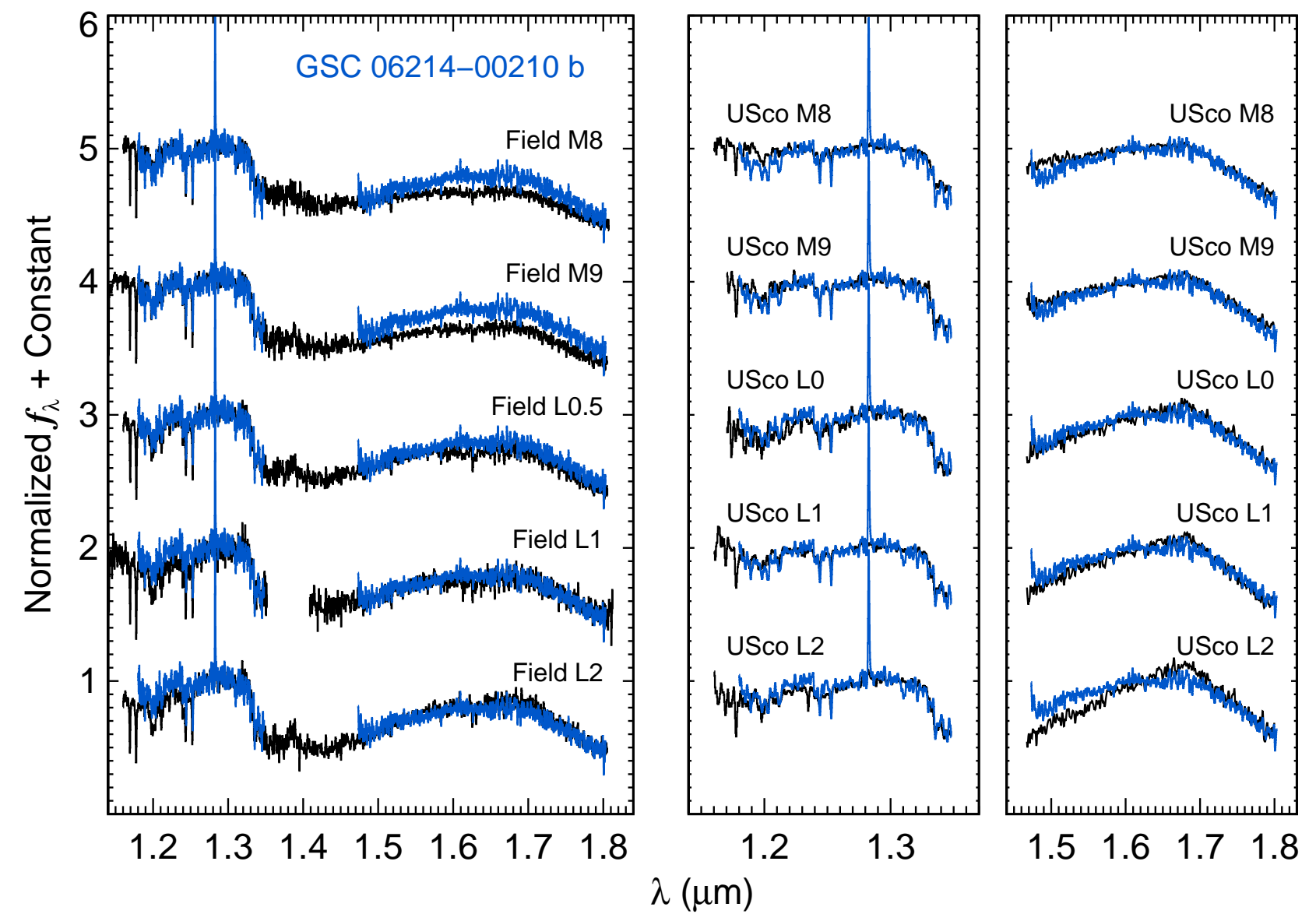

FIG. 3.- GSC 06214-00210 b (blue) compared to M8-L2 field objects (left panel) and members of Upper Scorpius (right panels). GSC 06214-00210 b resembles the L0.5 field object, although the depth of the K I lines are noticeably weaker in the younger object. The $J$ band of GSC 06214-00210 b is well matched to M9-L2 USco objects and the $H$ band to M9-L1 members; we adopt a spectral type of L0 \pm 1 . Field objects are from the IRTF Spectral Library (Ravner et al. 2009). From top to bottom they are Gl 752 B (M8), DENIS-P J1048-3956 (M9), 2MASS J0746+2000AB (L0.5), 2MASS J0208+2542 (L1), and Kelu-1 AB (L2). The USco spectra are from Lodieu et al. (2008). From top to bottom they are USco J155419-213543 (M8), USco J160847-223547 (M9), USco J160737-224247 (L0), USco J160723-221102 (L1), and USco J160603-221930 (L2). In the left panel the spectrum of GSC 06214-00210 b was smoothed to match the resolving power of the field objects $(R \sim 2000)$. In the right panels the spectrum of GSC 06214-00210 b and the USco spectra were smoothed to $R \sim 1000$. The spectra are normalized to $1.29-1.32 \mu \mathrm{m}$ in the left and middle panels and to $1.60-1.65 \mu \mathrm{m}$ in the right-most panel. 



FIG. 4.- BT-Settl-2010 atmospheric model fits (red) to our flux calibrated OSIRIS spectrum (black). $J$ (top), $H$ (middle), and joint $J+H$ (bottom) segments are fit separately; the best-fitting models are labeled. Contour plots map the average reduced $\chi^{2}$ values and inferred radii for each spectral segment based on Monte Carlo simulations of the data, which account for spectral measurement and photometric errors. We exclude the $\mathrm{Pa} \beta$ emission line in the fits (gray shaded region). Surface gravity is poorly constrained compared to effective temperature. The inferred temperature of $2700-3000 \mathrm{~K}$ is significantly higher than evolutionary-model predictions of $\sim 2200 \mathrm{~K}($ Figure 5 ). The reduced $\chi^{2}$ contours represent values of $8,10,15$, and 20 , and the radius contours represent values of $1.1,1.2,1.5$, and $1.8 R_{\text {Jup }}$. The best-fit model is plotted as a yellow star. Note that the best-fitting model to the joint $J+H$ spectrum is at the edge of the grid. 

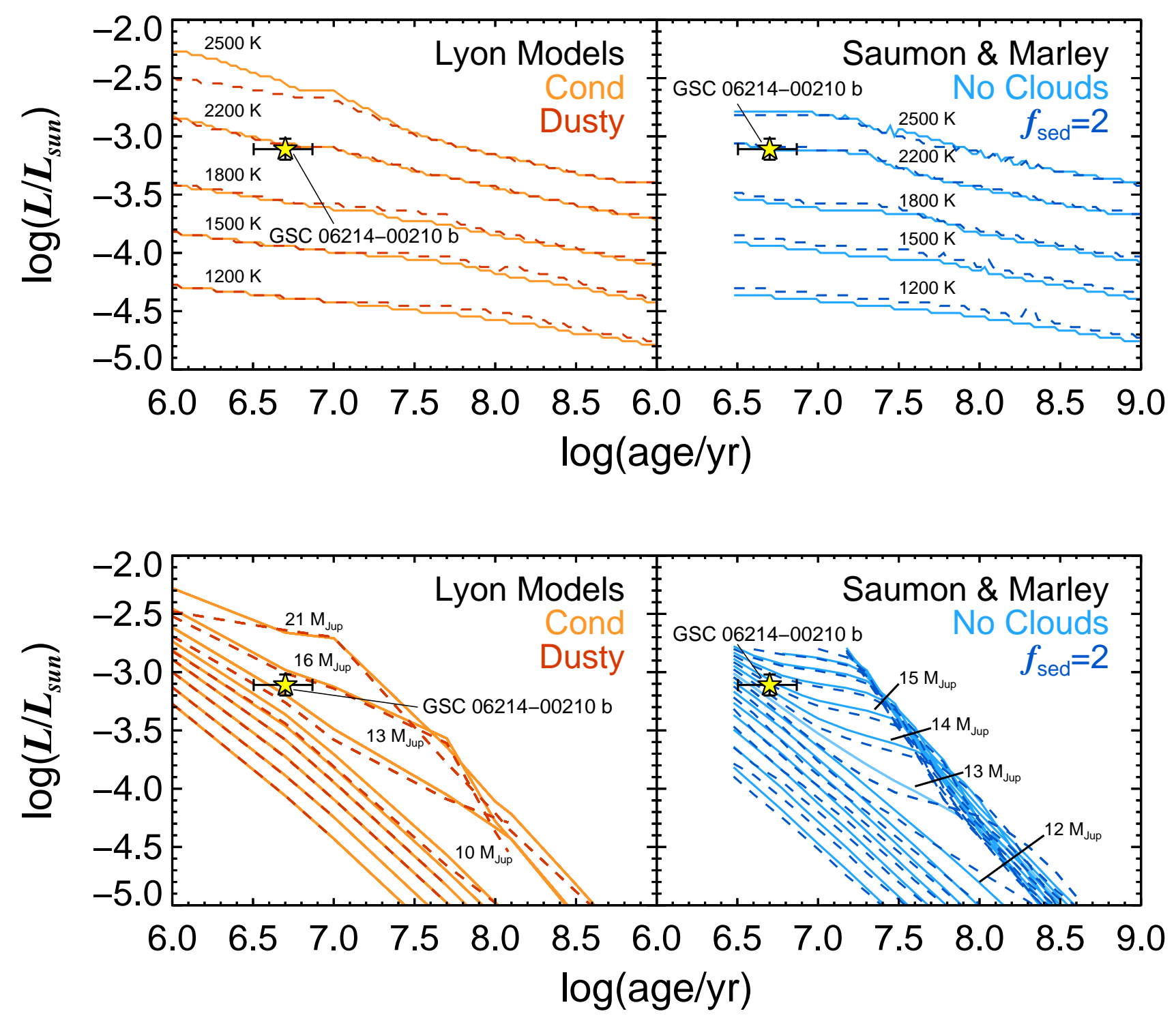

FIG. 5.- Evolutionary model-predicted temperature (top) and mass (bottom) of GSC 06214-00210 b. The left panels shows the Lyon Cond and Dusty models of Baraffe et al. (2003) and Chabrier et al. (2000), while the right panels shows the Saumon \& Marlev (2008) clear and cloudy $\left(f_{\text {sed }}=2\right)$ variants. In the top panels the models are interpolated onto a grid of constant temperatures. All models yield an effective temperature of $\sim 2200 \pm 100 \mathrm{~K}$ for GSC 06214-00210 b with little variation between the cloudy and clear prescriptions or the different models. The Lyon models and the Saumon \& Marley models predict masses within $1 M_{\mathrm{Jup}}$ from each other, with the cloudy versions yielding slightly higher masses than the clear variants. We adopt a mass of $14 \pm 2 M_{\mathrm{Jup}}$ for GSC 06214-00210 b. 


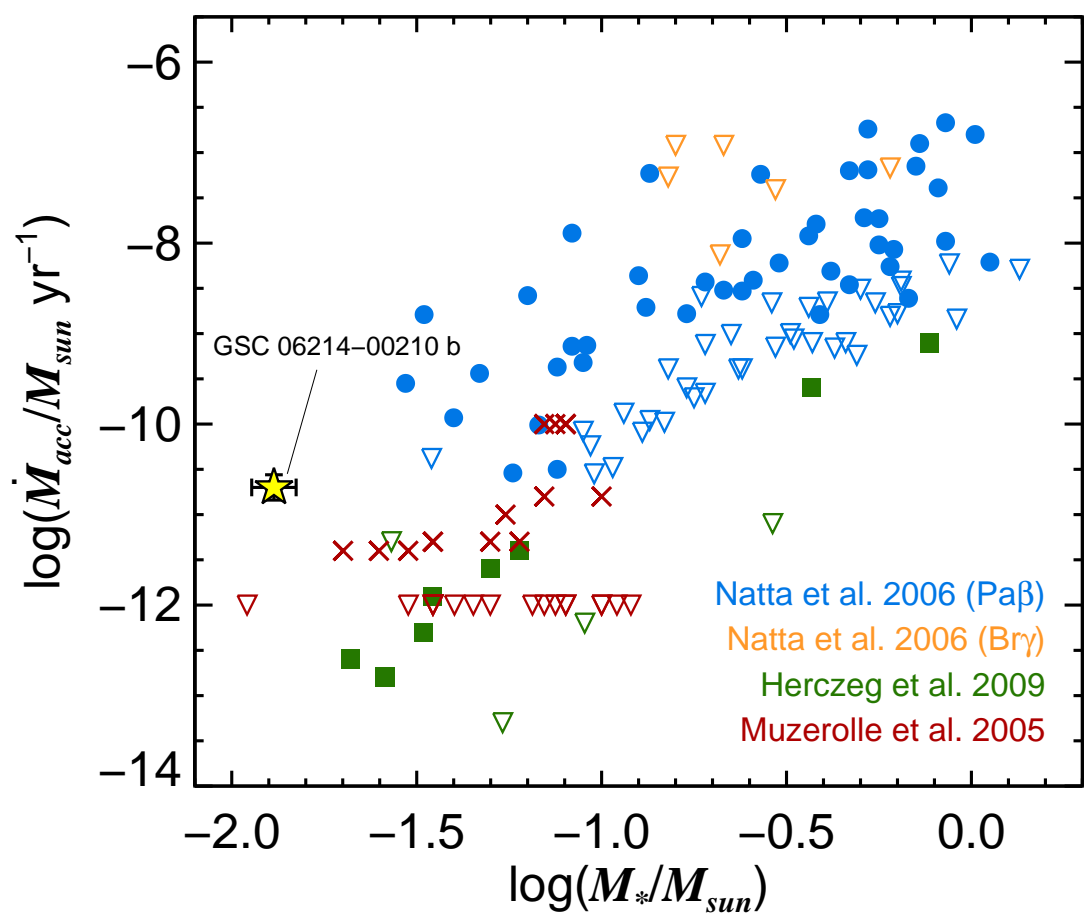

Fig. 6. - Mass accretion rates $(\dot{M})$ versus stellar mass. The data are from Natta et al. (2006, accretion rates derived using Pa $\beta$ lines are shown in blue while those using Br $\gamma$ are in orange), Herczeg et al. (2009, green), and Muzerolle et al. (2005, red). Open inverted triangles represent upper limits. Although the various samples represent different ages and methodologies used to derive mass accretion rates, the data clearly show the strong dependency of accretion rate on mass. The accretion rate of GSC 06214-00210 b appears to be somewhat higher than for low-mass brown dwarfs. The uncertainties shown here in the accretion rate for GSC 06214-00210 b ignore errors in the conversion from $L_{\mathrm{Pa} \beta}$ to $L_{\mathrm{acc}}$ from Natta et al. (2004) since we are making a direct comparison to their sample. The uncertainty increases to \pm 1.3 dex when the errors are included. 


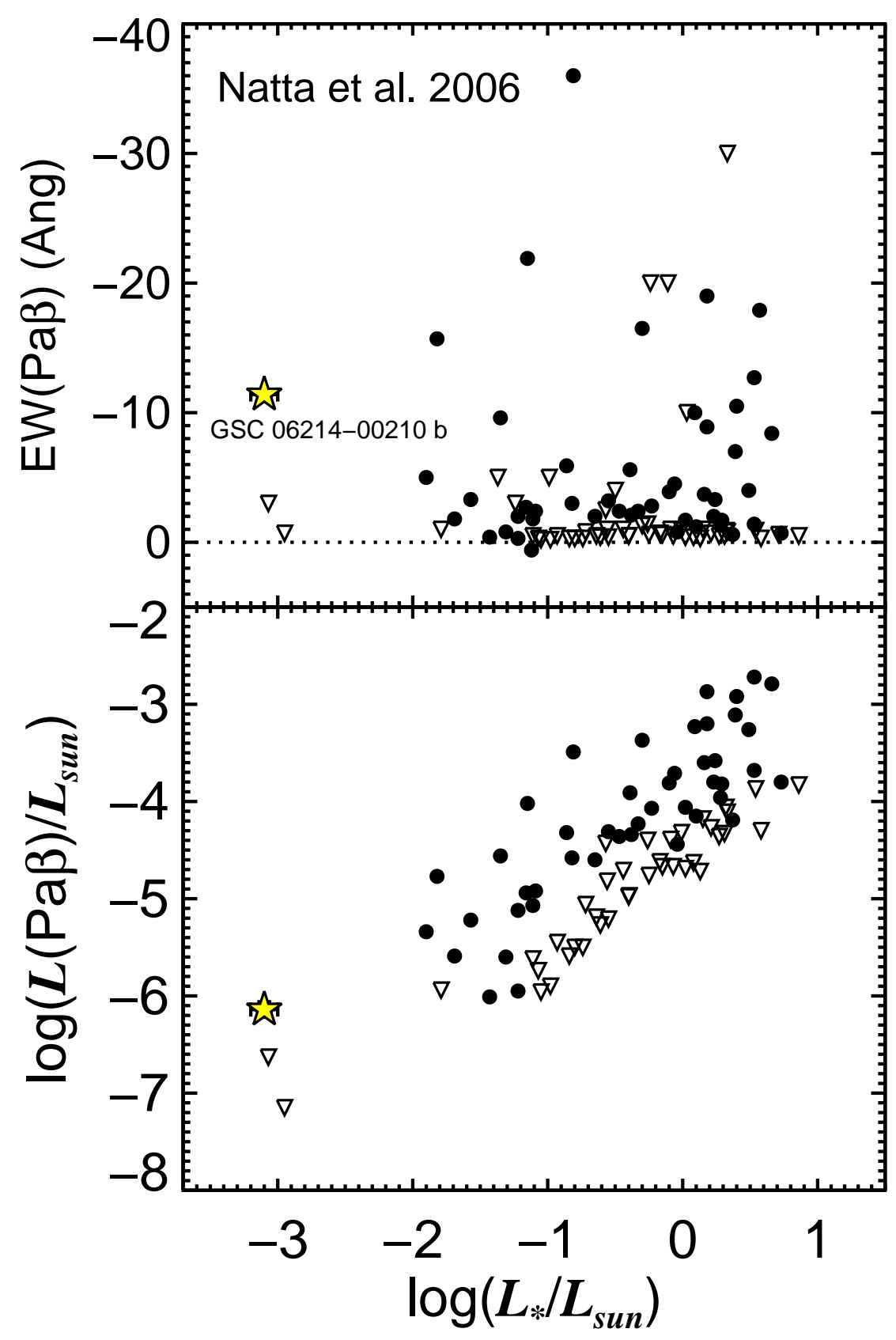

FIG. 7.- Comparison of the $\mathrm{Pa} \beta E W$ (top) and line luminosity (bottom) for GSC 02614-00210 b to the Natta et al. (2006) sample. Filled circles represent detections and open inverted triangles represent upper limits. The position of GSC 02614-00210 b is marked with a yellow star. 


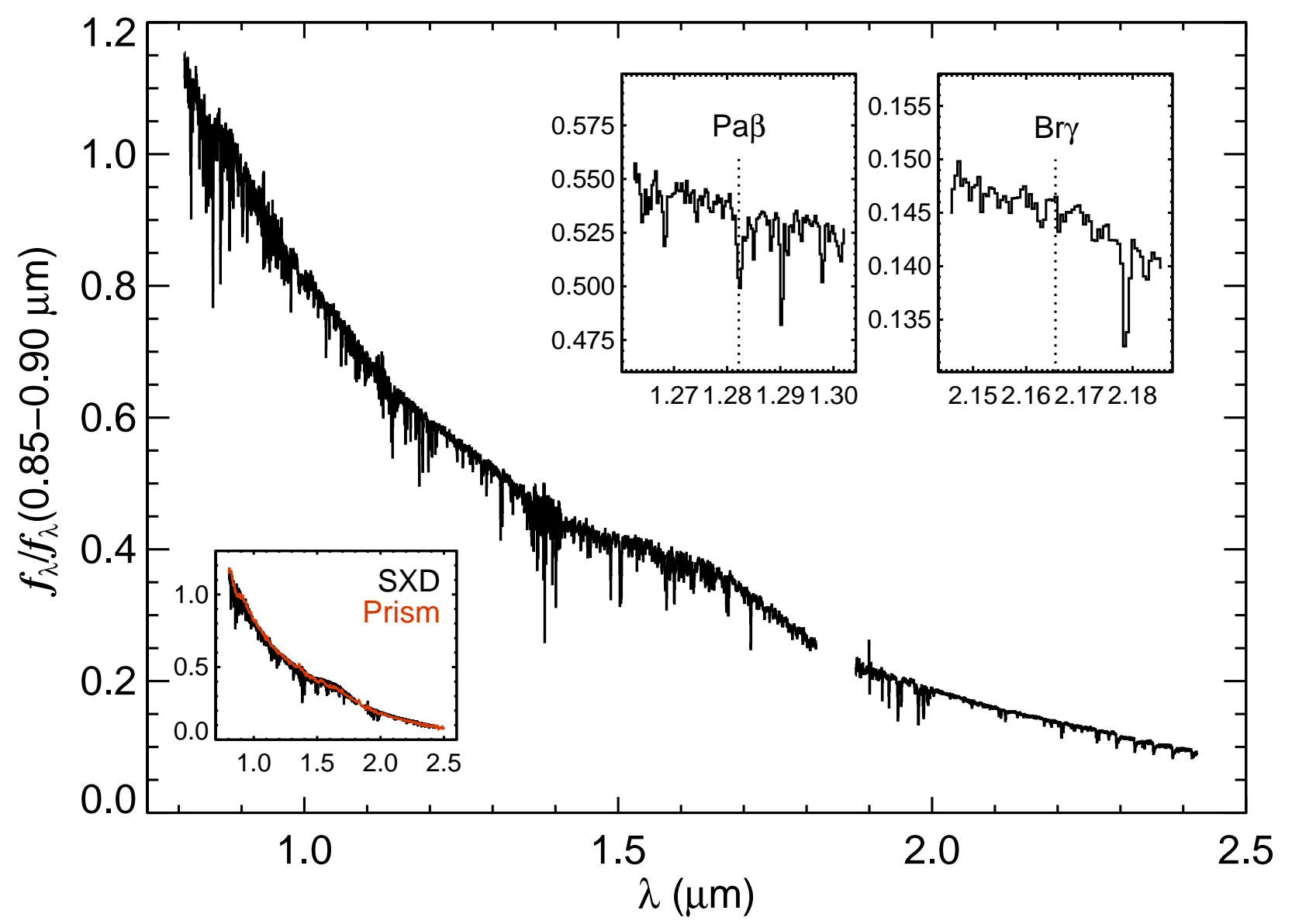

FIG. 8. - SXD spectrum of the primary GSC 06214-00210. No $\mathrm{Pa} \beta$ or $\mathrm{Br} \gamma$ emission lines are evident (upper right insets), which is consistent with mid-infrared photometry showing no indication of a disk. The bottom left inset shows that the SpeX/prism low resolution spectrum (red) agrees well with the SpeX/SXD moderate resolution data. 

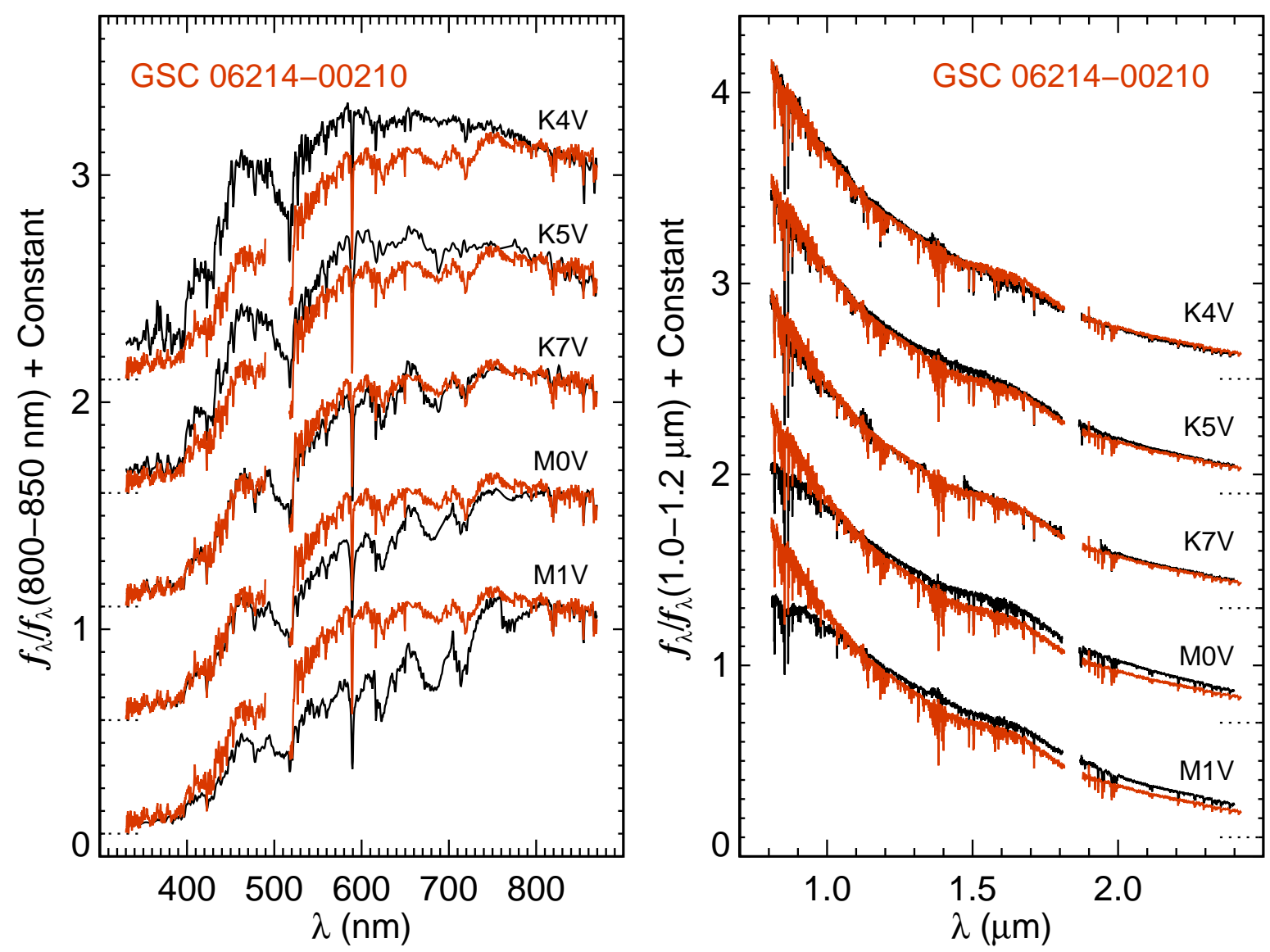

FIG. 9. - Left: UH 2.2m/SNIFS spectrum of the primary (red) compared to dwarf stars from the Pickles (1998) spectral library. The SNIFS spectrum is a composite of blue $(3300-4900 \AA)$ and red $(5170-8700 \AA)$ channels. GSC 06214-00210 is well matched by the K7 template. Right: SXD spectrum (red) compared to dwarf stars from the IRTF Spectral Library (Ravner et al. 2009). From top to bottom the objects are HD 45977 (K4), HD 36003 (K5), HD 201092 (K7), HD 19305 (M0), and HD 42581 (M1). K4-K7 objects are good matches to our near-infrared spectrum of the primary. We adopt a spectral type of K7 \pm 0.5 for GSC 06214-00210. 




FIG. 10.- Spectral energy distributions of GSC 06214-00210 and its companion. The $T_{\text {eff }}=4200 \mathrm{~K} / \log g=4.0$ Phoenix-Gaia model match the $0.6-12 \mu \mathrm{m}$ photometry of the primary. For the companion the $T_{\text {eff }}=2700 \mathrm{~K} / \log g=4.0$ and $T_{\text {eff }}=2200 \mathrm{~K} / \log g=4.0 \mathrm{BT}$-Settl-2010 models are plotted. The warmer temperature is from fitting atmospheric models and the cooler temperature is the evolutionary model prediction. Both models are flux calibrated to the $J$ band photometry of the companion. The excess flux in $L^{\prime}$, and perhaps also in $K$ are likely caused by thermal emission from a circumplanetary disk. Uncertainties in the photometry are smaller than the symbol sizes except for the WISE $22 \mu \mathrm{m}$ point. The slight excess at $22 \mu \mathrm{m}$ seen in the primary may be caused by a circumstellar disk. 


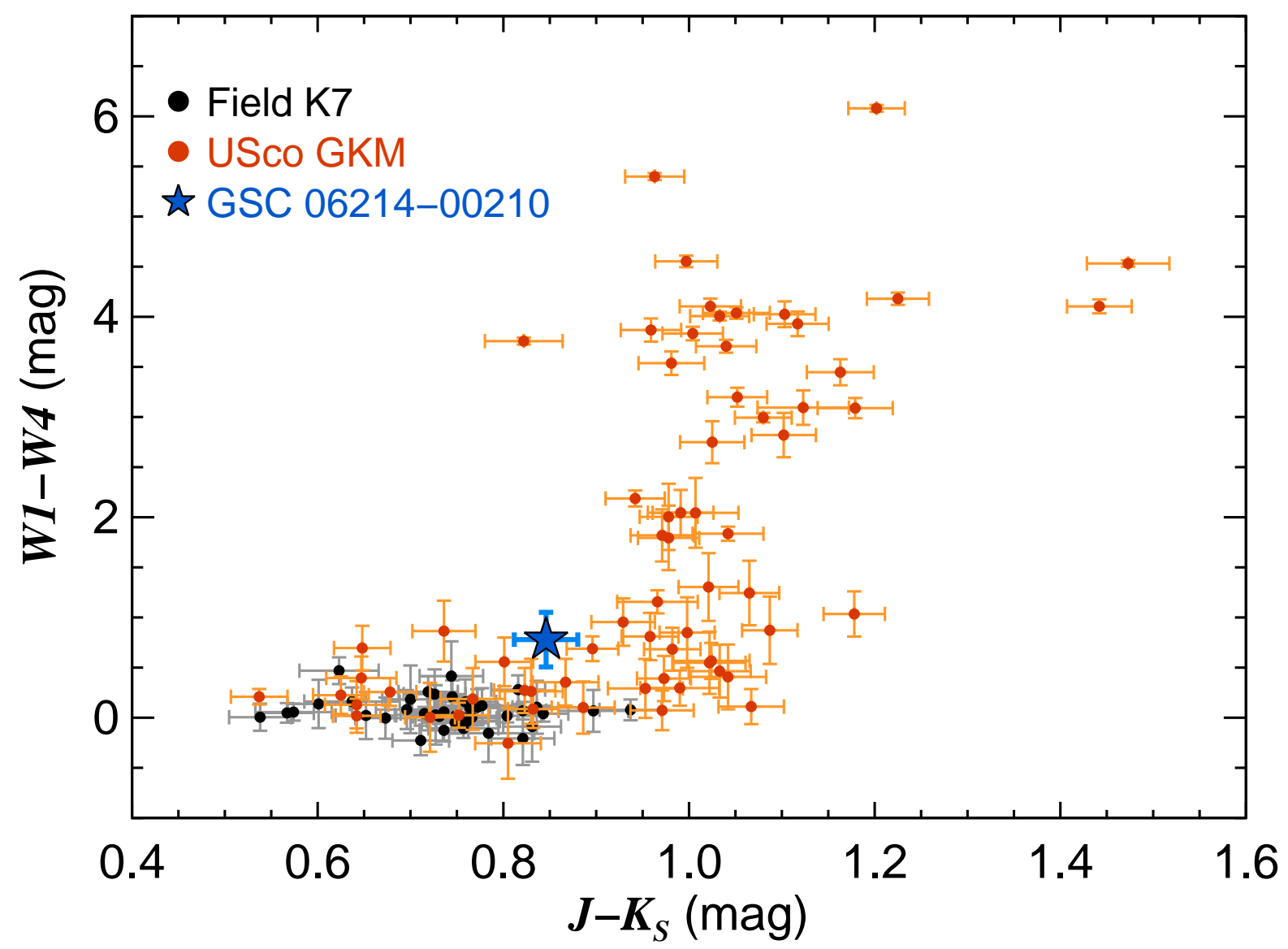

FIG. 11.-W $W-W 4$ vs $J-K_{S}$ diagram for field $\mathrm{K} 7$ stars (black filled circles) and Upper Scorpius members with spectral types of G, K, and $\mathrm{M}$ (filled red circles). Field stars have $W 1-W 4$ colors near 0.0 mag, while many of the redder USco members show an excess at these wavelengths. WISE photometry of GSC 06214-00210 (blue star) suggests an excess at the 2- $\sigma$ significance level. 


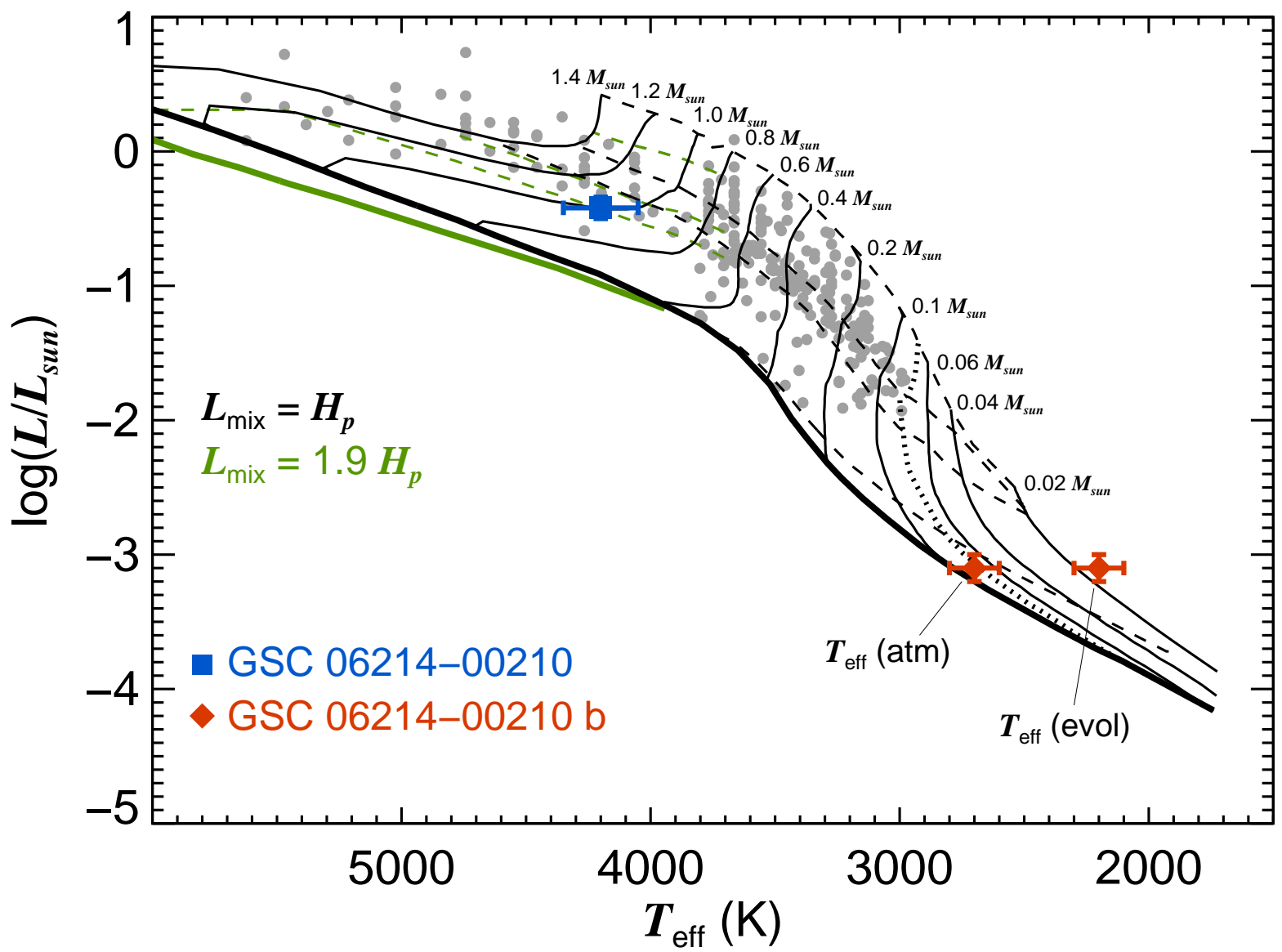

Fig. 12. - HR diagram for GSC 06214-00210 and its companion. The evolutionary models of Baraffe et al. (1998) with $L_{\mathrm{mix}}=H_{p}($ black) are overplotted with $1,5,10$, and $100 \mathrm{Myr}$ isochrones (dashed lines) and iso-mass tracks (solid lines) from $1.4-0.02 M_{\odot}$. The $1 \mathrm{Gyr}$ main sequence isochrone is shown as a thick line and the hydrogen burning minimum mass (HBMM) of $\sim 0.072 M_{\odot}$ is shown as a dotted line. The $L_{\mathrm{mix}}=1.9 H_{p}$ isochrones for $1 \mathrm{Myr}, 5 \mathrm{Myr}, 10 \mathrm{Myr}$, and $1 \mathrm{Gyr}$ are shown in green. The position of the primary indicates a mass of $\sim 1 M_{\odot}$; our adopted mass of $0.9 \pm 0.1 M_{\odot}$ is based on an average from several evolutionary models (see text for details). The position of GSC 06214-00210 b (red) is shown for the warmer atmospheric model-inferred temperature $(2700 \mathrm{~K})$ and the cooler evolutionary modelderived temperature $(2200 \mathrm{~K})$. The warmer temperature is inconsistent with the young age of the system. Gray circles show members of Upper Scorpius from Preibisch \& Mamaiek (2008). 


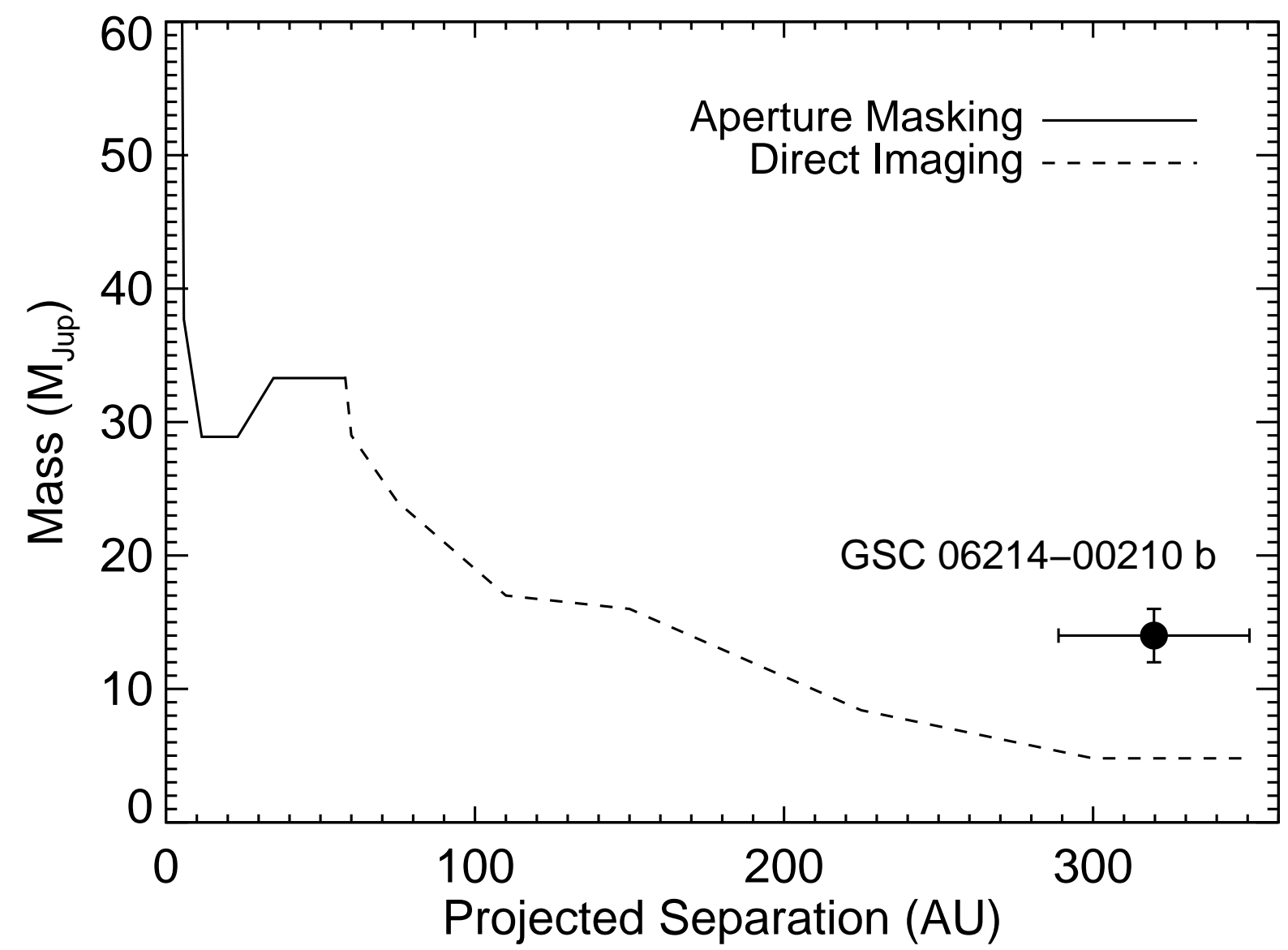

FIG. 13. - Constraints on additional companions around GSC 06214-00210 based on aperture masking results from Kraus et al. (2008) and direct imaging from Ireland et al. (2011). Objects with masses twice that of GSC 06214-00210 b are excluded at projected separations $\gtrsim 60 \mathrm{AU}$. The aperture masking limits exclude brown dwarfs with masses $\gtrsim 35 M_{\mathrm{Jup}}$ at projected separations $\gtrsim 5$ AU. The uncertainty in the projected separation of GSC 06214-00210 b (30 AU) is dominated by the distance error. 